\title{
An Edgeworth expansion for finite- population $U$-statistics
}

\author{
MINDAUGAS BLOZNELIS ${ }^{1}$ and FRIEDRICH GÖTZE ${ }^{2}$ \\ ${ }^{1}$ Department of Mathematics, Vilnius University, Naugarduko 24, Vilnius 2006, Lithuania. \\ E-mail: mindaugas.bloznelis@maf.vu.lt \\ ${ }^{2}$ Fakultät für Mathematik, Universität Bielefeld, Postfach 100131, 33501 Bielefeld, Germany. \\ E-mail: goetze@mathematik.uni-bielefeld.de
}

Suppose that $U$ is a $U$-statistic of degree 2 based on $N$ random observations drawn without replacement from a finite population. For the distribution of a standardized version of $U$ we construct an Edgeworth expansion with remainder $O\left(N^{-1}\right)$ provided that the linear part of the statistic satisfies a Cramér type condition.

Keywords: central limit theorem; Edgeworth expansion; finite population; $U$-statistic; sampling without replacement

\section{Introduction and results}

Let $\mathscr{b}=\left\{a_{1}, \ldots, a_{n}\right\}$ denote a population of size $n$ and let $\mathscr{H}: \mathscr{b} \times \mathscr{b} \rightarrow \mathbb{R}$ denote symmetric function of its two arguments. By $X_{1}, \ldots, X_{N}, N \leqslant n$, we denote random variables with values in $\mathscr{C}$ such that $X=\left\{X_{1}, \ldots, X_{N}\right\}$ represents a random sample from $\mathscr{C}$ of size $N$ drawn without replacement, i.e. $\mathrm{P}\{X=B\}=\left(\begin{array}{c}n \\ N\end{array}\right)^{-1}$ for any subset $B \subset \mathscr{A}$ of size $N$. We shall investigate the second-order asymptotics of the distribution of the statistic

$$
U=\sum_{1 \leqslant i \leqslant j \leqslant N} \mathscr{H}\left(X_{i}, X_{j}\right)
$$

We assume that the statistic is centred. Write

$$
U=L+Q
$$

where

$$
L=\sum_{i=1}^{N} g_{1}\left(X_{i}\right) \text { and } Q=\sum_{1 \leqslant i \leqslant j \leqslant N} g_{2}\left(X_{i}, X_{j}\right)
$$

are respectively the linear and the quadratic part of the statistic. Here

$$
g_{1}(x)=(N-1) t(x), \quad t(x)=\frac{n-1}{n-2} \mathrm{E}\left(\mathscr{H}\left(X_{1}, X_{2}\right) \mid X_{1}=x\right)
$$

and 


$$
g_{2}\left(x_{1}, x_{2}\right)=\mathscr{H}\left(x_{1}, x_{2}\right)-t\left(x_{1}\right)-t\left(x_{2}\right)
$$

Since

$$
\mathrm{E}\left(g_{2}\left(X_{1}, X_{2}\right) \mid X_{1}=x\right)=0, \quad \text { for all } x \in \mathscr{A},
$$

the random variables $g_{1}\left(X_{i}\right)$ and $g_{2}\left(X_{j}, X_{k}\right), 1 \leqslant i \leqslant N, 1 \leqslant j<k \leqslant N$ (and thus $L$ and $Q$ ) are uncorrelated. If the linear part $L$ dominates the statistic, for large $N$, the distribution of $U$ can be approximated by a Gaussian distribution using the central limit theorem (CLT).

The asymptotic normality of linear statistics based on samples drawn without replacement from finite populations has been studied by a number of authors. Erdós and Rényi (1959) proved the CLT under very mild conditions. The rate of convergence in the CLT was first studied by Bikelis (1969). Berry-Esseen bounds of order $O\left(N^{-1 / 2}\right)$ were obtained by Höglund (1978). Robinson (1978) proved the validity of an Edgeworth expansion with a remainder of order $O\left(N^{-3 / 2}\right)$; see also Bickel and van Zwet (1978).

Nandi and Sen (1963) studied the asymptotic behaviour of finite-population $U$-statistics and showed that under proper regularity conditions the sequence of distributions of normalized $U$-statistics converges to the standard normal distribution. The rate of this convergence was investigated by Zhao and Chen (1987; 1990), Kokic and Weber (1990; 1991) and, as a particular case of the rate of convergence of general multivariate sampling statistics, by Bolthausen and Götze (1993). In the case of independent and identically distributed (i.i.d.) observations the second-order asymptotic theory has been developed for U-statistics: see Bickel (1974), Götze (1979), Callaert et al. (1980), Bickel et al. (1986) and, for more general asymptotically normal symmetric statistics, Bentkus et al. (1997). In contrast to the independent case, there are only a few results concerned with higher-order asymptotics of nonlinear finite population statistics. Babu and Singh (1985) proved the validity of an Edgeworth expansion with a remainder $o\left(N^{-1 / 2}\right)$ for finite-population multivariate sample mean and applied this result to establish expansions for statistics that can be represented as smooth functions of multivariate sample means, e.g. Student's $t$. Kokic and Weber (1990) established a one-term Edgeworth expansion with the remainder $o\left(N^{-1 / 2}\right)$ for finite-population $U$-statistics of degree 2 .

By way of comparison to the results described above, we shall provide an explicit remainder term of order $O\left(N^{-1}\right)$ for finite population $U$-statistics which is optimal assuming a Cramér condition on the linear term only. The proof is based on a finitepopulation variant of Hoeffding's decomposition as well as the Erdós-Rényi representation and some ideas due to Bentkus et al. (1997) such as data-dependent smoothing.

Assume that

$$
\sigma^{2}=N \mathrm{Eg}_{1}^{2}\left(X_{1}\right)>0 .
$$

The distribution function of the standardized statistic, $F(x)=\mathrm{P}\{U \leqslant x \sigma\}$, will be approximated by the one-term Edgeworth expansion,

$$
G(x)=\Phi\left(\frac{x}{\sqrt{q}}\right)-\frac{(q-p) q^{-1 / 2} \alpha+3 q^{1 / 2} \kappa}{6 \sigma^{3} N^{1 / 2}} \Phi^{\prime \prime \prime}\left(\frac{x}{\sqrt{q}}\right) .
$$

Here $\Phi(x)$ is the standard normal distribution function, 


$$
p=N / n, \quad q=1-p
$$

and

$$
\alpha=N^{3 / 2} \mathrm{Eg}_{1}^{3}\left(X_{1}\right), \quad \kappa=N^{5 / 2} \mathrm{Eg}_{2}\left(X_{1}, X_{2}\right) g_{1}\left(X_{1}\right) g_{1}\left(X_{2}\right) .
$$

We shall derive bounds for the remainder

$$
\Delta=\sup _{x \in \mathbb{R}}|F(x)-G(x)| .
$$

To prove the validity of an Edgeworth expansion, i.e. to establish bounds for $\Delta$, in addition to moment conditions one needs to impose a smoothness condition, cf. Bickel and Robinson (1982). For instance, in the classical case of standardized sums $S=$ $\left(Y_{1}+\ldots+Y_{N}\right) / \sqrt{N}$ of i.i.d. random variables $Y_{1}, \ldots, Y_{N}$ such that $\mathrm{E} Y_{1}=0, \mathrm{E} Y_{1}^{2}=1$ and $\mathrm{E} Y_{1}^{4}<\infty$, asymptotic expansions for the distribution $F_{S}$ of $S$ with remainder $O\left(N^{-1}\right)$ are obtained assuming Cramér's condition,

$$
\sup _{|t|>a}\left|\operatorname{E} \exp \left\{\mathrm{i} t Y_{1}\right\}\right|<1 .
$$

Bentkus et al. (1997) introduced a local version of Cramér's condition (C), namely,

$$
\rho_{Y_{1}}(a, b):=1-\sup _{a \leqslant|t| \leqslant b}\left|\operatorname{E} \exp \left\{\mathrm{i} t Y_{1}\right\}\right|>0 .
$$

Condition $\left(\mathbf{C}^{\prime}\right)$ (with $a=1 / \mathrm{E}\left|Y_{1}\right|^{3}$ and $b=N^{1 / 2}$ ) is somewhat weaker than $(\mathbf{C})$ but still sufficient to prove the validity of Edgeworth expansions for $F_{S}$ up to order $O\left(N^{-1}\right)$. This modification is useful in more general situations, where $Y_{1}$ depends on $N$ in an implicit way; see Bentkus et al. (1997).

For a sufficiently small absolute constant $b_{1}$, say $b_{1}=0.0001$, we shall assume that the distribution of the random variable $Z=\sqrt{N} g_{1}\left(X_{1}\right) / \sigma$ satisfies condition $\left(\mathbf{C}^{\prime}\right)$ with $a^{\prime}=b_{1} / \mathrm{E}|Z|^{3}$ and $b^{\prime}=N^{1 / 2}$, i.e.

$$
\rho=\rho_{Z}\left(a^{\prime}, b^{\prime}\right)>0
$$

Write, for $r=1,2, \ldots$,

$$
\beta_{r}=\mathrm{E}\left|N^{1 / 2} g_{1}\left(X_{1}\right)\right|^{r} \quad \text { and } \quad \gamma_{r}=\mathrm{E}\left|N^{3 / 2} g_{2}\left(X_{1}, X_{2}\right)\right|^{r} .
$$

Then the following estimate holds for the remainder $\Delta$.

Theorem 1.1. There exists an absolute constant $A>0$ such that

$$
\sup _{x \in \mathbb{R}}|F(x)-G(x)| \leqslant \frac{A}{N} \frac{\beta_{4}+\gamma_{4}}{\rho^{2} q^{2} \sigma^{4}} .
$$

For linear statistics we obtain the following result.

Theorem 1.2. There exists an absolute constant $B>0$ such that 


$$
\left|\mathrm{P}\{L \leqslant x\}-\Phi\left(\frac{x}{\sqrt{q}}\right)+\frac{(q-p) q^{-1 / 2} \alpha}{6 \sigma_{3} N^{1 / 2}} \Phi^{\prime \prime \prime}\left(\frac{x}{\sqrt{q}}\right)\right| \leqslant \frac{B}{N} \frac{\beta_{4}}{\rho^{2} q \sigma^{4}}
$$

The estimates in Theorems 1.1 and 1.2 hold for any fixed sample size $N$, population size $n$ and functions $\mathscr{H}$. If $\beta_{4} / \sigma^{4}$ and $\gamma_{4} / \sigma^{4}$ are bounded and $q$ and $\rho$ are bounded away from 0 and $N \rightarrow \infty$ and $n \rightarrow \infty$, then these results establish Edgeworth expansions with the remainder $O\left(N^{-1}\right)$.

The case where $n \rightarrow \infty$ and $N$ is fixed corresponds to the i.i.d. situation. By the law of large numbers we obtain a corollary for independent observations. Let $\mathscr{E}$ denote a measurable space and let $\mathscr{C}_{1}, \mathscr{C}_{2}, \ldots$ be i.i.d. random variables with values in $\mathscr{E}$. Write

$$
\tilde{U}=\sum_{1 \leqslant i \leqslant j \leqslant N} \mathscr{H}\left(\mathscr{B}_{i}, \mathscr{C}_{j}\right) .
$$

Here $\mathscr{H}: \mathscr{E} \times \mathscr{E} \rightarrow \mathbb{R}$ denotes a measurable function symmetric in its two arguments such that $\mathrm{E} \mathscr{H} \mathscr{C}^{2}\left(\mathscr{C}_{1}, \mathscr{C}_{2}\right)<\infty$. We assume that $\mathrm{E} \tilde{U}=0$ and decompose

$$
\tilde{U}=\sum_{i=1}^{N} \tilde{g}_{1}\left(X_{i}\right)+\sum_{1 \leqslant i \leqslant j \leqslant N} \tilde{g}_{2}\left(X_{i}, X_{j}\right) .
$$

Here $\tilde{g}_{1}$ and $\tilde{g}_{2}$ are defined in the same way as $g_{1}$ and $g_{2}$, but using $\tilde{t}(x)=$ $\mathrm{E}\left(\mathscr{H}\left(\mathscr{C}_{1}, \mathscr{C}_{2}\right) \mid \mathscr{C}_{1}=x\right)$ instead of $t(x)$. Let $\tilde{\sigma}, \tilde{\alpha}, \tilde{\beta}_{k}, \tilde{\gamma}_{k}, k=2,3,4$, and $\tilde{\kappa}$ denote the moments of $\tilde{g}_{1}\left(\mathscr{C}_{1}\right)$ and $\tilde{g}_{2}\left(\mathscr{C}_{1}, \mathscr{C}_{2}\right)$ corresponding to $\sigma, \alpha, \beta_{k}, \gamma_{k}$ and $\kappa$. We shall assume that

$$
\tilde{\rho}=\rho_{\mathscr{\&}}(\tilde{a}, \tilde{b})>0, \quad \mathscr{Z}=\sqrt{N} \tilde{g}_{1}\left(\mathscr{B}{ }_{1}\right) / \tilde{\sigma},
$$

where $\tilde{a}=b_{1} / \mathrm{E}|\mathscr{Z}|^{3}$ and $\tilde{b}=\sqrt{N}$. Then we have:

Corollary 1.3. There exists an absolute constant $A>0$ such that

$$
\left|\mathrm{P}\{\tilde{U} \leqslant \tilde{\sigma} x\}-\Phi(x)+\frac{\tilde{\alpha}+3 \tilde{\kappa}}{6 \tilde{\sigma}^{3} N^{1 / 2}} \Phi^{\prime \prime \prime}(x)\right| \leqslant \frac{A}{N} \frac{\tilde{\beta}_{4}+\tilde{\gamma}_{4}}{\tilde{\rho}^{2} \tilde{\sigma}^{4}} .
$$

Hence Theorem 1.1, which yields this result as a special case, may be regarded as a partial extension of the result of Bentkus et al. (1997) to a simple random sampling model. They proved the validity of an Edgeworth expansion with remainder $O\left(N^{-1}\right)$ for general symmetric asymptotically normal statistics based on i.i.d. observations. In the case of $U$-statistics of degree 2 their result yields the estimate as in Corollary 1.3 but with a lower moment $\tilde{\gamma}_{3} / \tilde{\sigma}^{3}$ instead of $\tilde{\gamma}_{4} / \tilde{\sigma}^{4}$ in the remainder.

An example given in Theorem 1.4 of Bentkus et al. (1997) shows that a Cramér type condition on the linear part and the existence of moments of arbitrarily high order of the linear and quadratic parts of the statistic (based on i.i.d. observations) are not sufficient to obtain higher-order approximations (those with remainders $o\left(N^{-1}\right)$ ) to the distribution function of $U$. Hence, in this sense Corollary 1.3 and thus Theorem 1.1 are the best possible. To prove the validity of an Edgeworth expansion with remainder $o\left(N^{-1}\right)$, one 
needs in addition to impose a smoothness condition on the distribution of the quadratic part; see, for example, Bickel et al. (1986).

Let us compare our results with those of Robinson (1978) and Kokic and Weber (1990). Robinson (1978) proved the validity of a two-term Edgeworth expansion with remainder $O\left(N^{-3 / 2}\right)$ for linear statistics like $L$ in (1.1) assuming the following Cramér type condition. This condition, first used in Albers et al. (1976), requires for a random variable $Z$ that there exists an $\varepsilon>0$ such that

$$
\tau_{Z}(\varepsilon, a, b)=1-\sup _{s \in \mathbb{R}, a \leqslant|t| \leqslant b} \mathrm{P}\left\{t Z \in \mathscr{D}^{\varepsilon}+s\right\}>0 .
$$

Here $\mathscr{B}=\{2 \pi r, r=0, \pm 1, \pm 2, \ldots\}$ and $\mathscr{B}^{\varepsilon}$ denotes the $\varepsilon$-neighbourhood of a set $\mathscr{B} \subset \mathbb{R}$. Notice that $\varepsilon_{1} \leqslant \varepsilon_{2}$ implies $\tau_{Z}\left(\varepsilon_{1}, a, b\right) \geqslant \tau_{Z}\left(\varepsilon_{2}, a, b\right)$. Robinson assumed that given $C^{\prime}>0$

$$
\text { there exist } \varepsilon, \delta>0 \text { and } C>0 \text { such that } \tau_{Z}(\varepsilon, a, b)>\delta \text {, }
$$

for

$$
Z=\sqrt{N} g_{1}\left(X_{1}\right) / \sigma, \quad a^{-1}=\max _{1 \leqslant i \leqslant n}\left|z_{i}\right| / C^{\prime}, \quad b^{-1}=p \mathrm{E}|Z|^{5} /(C N) .
$$

Here $\left\{z_{1}, \ldots, z_{n}\right\}$ denotes the set of values of the random variable $Z$. Note that $\max _{i}\left|z_{i}\right|=\max _{i}\left|z_{i}\right| \mathrm{E} Z^{2} \geqslant \mathrm{E}|Z|^{3}$, because of $\mathrm{E} Z^{2}=1$. For a sequence of finite-population linear statistics, say $\left(L_{n}\right)$, Robinson's (1978) theorem establishes an Edgeworth expansion with remainder $O\left(N^{-3 / 2}\right)$ provided that $\beta_{5} / \sigma^{5}$ is bounded, $p$ and $q$ are bounded away from 0 and (1.7) holds with $\varepsilon, \delta$ and $C$ not depending on $n$ as $n \rightarrow \infty$. Robinson's (1978) result was used by Kokic and Weber (1990) to show $\Delta=o\left(N^{-1 / 2}\right)$. The bounds for the remainders in these papers involve constants which implicitly depend on $p$.

In Section 2 we compare conditions (c) and (1.5). Proofs of Theorems 1.1 and 1.2 and of Corollary 1.3 are given in Sections 3 and 4. Auxiliary results are gathered together in Section 5.

\section{Smoothness conditions}

Modifications of Cramér's condition (C) that ensure the validity of Edgeworth expansions for sums of random variables assuming a finite number of values only were considered by Albers et al. (1976), van Zwet (1982), Does (1983) and Schneller (1989); see also Bickel and Robinson (1982). In this section we show that a Cramér type condition used in Albers et al. (1976) and Robinson (1978) is equivalent to that introduced in Bentkus et al. (1997) namely, that the conditions (1.5) and (c) are equivalent. More specifically, given a random variable $Z$ and numbers $0<a<b,(1.5)$ implies $\tau_{Z}(\rho, a, b)>\rho / 4$. Furthermore, if (c) holds for some $\varepsilon>0$, then $\rho_{Z}(a, b)>\varepsilon^{2} \tau_{Z}(\varepsilon, a, b) / \pi^{2}$; see Lemma 2.1 below.

In order to check condition (c) one needs to maximize a bivariate function over the set $(s, t) \in[-\pi, \pi] \times\{a \leqslant|t| \leqslant b\}$. Such a (maximization) problem can be difficult to solve numerically. A symmetrization argument suggests a version of condition (c) which is easier to check. Let $Z^{\prime}$ denote an independent copy of $Z$ and let $Z^{*}=Z-Z^{\prime}$ denote a symmetrization of $Z$. The condition 
there exists $\varepsilon>0 \quad$ such that $\tau_{Z}^{*}(\varepsilon, a, b)=1-\sup _{a \leqslant|t| \leqslant b} \mathrm{P}\left\{t Z^{*} \in \mathscr{D}^{\varepsilon}\right\}>0$

requires the estimation of the maximum of an univariate function only. Condition ( $\left.\mathbf{c}^{*}\right)$ was proposed by $\mathrm{V}$. Bentkus. Notice that $\varepsilon_{1}<\varepsilon_{2}$ implies $\tau_{Z}^{*}\left(\varepsilon_{2}, a, b\right) \leqslant \tau_{Z}^{*}\left(\varepsilon_{1}, a, b\right)$. The following lemma shows that conditions $\left(\mathbf{c}^{*}\right)$ and (c) are equivalent. Write

$$
\delta_{Z}(a, b)=1-\sup \{\mathrm{E} \cos (t Z+s): s \in \mathbb{R}, a \leqslant|t| \leqslant b\} .
$$

Lemma 2.1 Let $Z$ be a random variable. For $0<a<b$ and $0<\varepsilon<\pi$, write

$\rho=\rho_{Z}(a, b), \quad \tau_{\varepsilon}=\tau_{Z}(\varepsilon, a, b) \quad \tau_{\varepsilon}^{*}=\tau_{Z}^{*}(\varepsilon, a, b), \quad u=\pi^{-1} \varepsilon \tau_{\varepsilon}^{*}, \quad v=\pi^{-1} \varepsilon \tau_{\varepsilon}$.

The following inequalities hold:

$$
\frac{\varepsilon^{2} \tau_{\varepsilon}}{\pi^{2}} \leqslant \rho \leqslant 4 \tau_{\rho}, \quad \frac{\varepsilon^{2} \tau_{\varepsilon}^{*}}{\pi^{2}} \leqslant \rho \leqslant 4 \tau_{\rho}^{*}, \quad \tau_{v}^{*} \geqslant \frac{\varepsilon^{2} \tau_{\varepsilon}}{2 \pi^{2}}, \quad \tau_{u} \geqslant \frac{\varepsilon^{2} \tau_{\varepsilon}^{*}}{4 \pi^{2}}, \quad \delta_{Z}(a, b) \geqslant \rho .
$$

The proof of Lemma 2.1 is elementary; see Bloznelis and Götze (1997).

\section{Proofs}

Throughout this section and the next we shall assume without loss of generality that $\beta_{2}=1$. Since the proof of our main result, Theorem 1.1 is rather complex and involved we shall first outline the various steps.

In the first step, choosing $m \approx \ln N$, we replace the statistic $U$ by

$$
U_{1}=L^{\prime}+U^{\prime}, \quad U^{\prime}=g_{1}\left(X_{m+1}\right)+\ldots+g_{1}\left(X_{N}\right)+\sum_{m+1 \leqslant i<j \leqslant N} g_{2}\left(X_{i}, X_{j}\right),
$$

where

$$
L^{\prime}=l\left(X_{1}\right)+\ldots+l\left(X_{m}\right)
$$

with

$$
l(x)=g_{1}(x)+l_{0}(x), \quad l_{0}(x)=\sum_{j=m+1}^{N} g_{2}\left(x, X_{j}\right),
$$

is a conditionally linear statistic given $X_{m+1}, \ldots, X_{N}$. Write

$$
F_{X}(x)=\mathrm{P}\left\{U_{1} \leqslant x \mid X_{m+1}, \ldots, X_{N}\right\}, \quad f_{1}(t)=\mathrm{E}\left(\exp \left\{\mathrm{i} t U_{1}\right\} \mid X_{m+1}, \ldots, X_{N}\right) .
$$

In the second step we construct upper/lower bounds for conditional probabilities

$$
\begin{aligned}
& F_{X}(x+) \leqslant \frac{1}{2}+\mathrm{VP} \int_{\mathbb{R}} \exp \{-\mathrm{i} x t\} \frac{1}{H} K\left(\frac{t}{H}\right) f_{1}(t) \mathrm{d} t, \\
& F_{X}(x-) \geqslant \frac{1}{2}-\mathrm{VP} \int_{\mathbb{R}} \exp \{-\mathrm{i} x t\} \frac{1}{H} K\left(\frac{-t}{H}\right) f_{1}(t) \mathrm{d} t,
\end{aligned}
$$


where $F(x+)=\lim _{z \downarrow x} F(z), F(x-)=\lim _{z \uparrow x} F(z)$ and VP denotes Cauchy's principal value (Prawitz's (1972) smoothing lemma). The bounded weight function $K(t / H)$, vanishing for $|t|>H$, and the cut-off $H=O(N)$ are specified below. Taking expectations of the left- and right-hand sides respectively, we obtain upper and lower bounds for the distribution function $F_{1}(x)=\mathrm{P}\left\{U_{1} \leqslant x\right\}$; see (3.7) and (3.8) below.

In the third step we construct a bound for the integral of $f_{1}(t) K(t / H)$ over the region $c N^{1 / 2} \leqslant|t| \leqslant H$. In the classical linear statistic case the bounds for the characteristic function for large values of $t$, like $c N^{1 / 2} \leqslant|t| \leqslant C N$, are implied by Cramér's condition (C). We write

$$
\left|f_{1}(t)\right| \leqslant\left|\mathrm{E}\left(\exp \left\{\mathrm{i} t\left(l\left(X_{1}\right)+\ldots+l\left(X_{m}\right)\right)\right\} \mid X_{m+1}, \ldots, X_{N}\right)\right|
$$

and show that the Cramér condition $\left|\operatorname{E} \exp \left\{\operatorname{itg}_{1}\left(X_{1}\right)\right\}\right|<1-\rho$ (we do not require $\left.\left|\mathrm{E} \exp \left\{\mathrm{i} t l\left(X_{1}\right)\right\}\right|<1-\rho\right)$, in combination with a suitable choice of the cut-off $H=H\left(X_{m+1}, \ldots, X_{N}\right)$ implies a bound like $\left|f_{1}\right| \leqslant(1-c \rho)^{m}$, for some $0<c<1$. The techniques are somewhat complicated by the fact that $X_{1}, \ldots, X_{m}$ are exchangeable only and we get the independence via the Erdös-Rényi decomposition for (conditional and unconditional) characteristic functions.

In the next step we interchange the conditional characteristic function with the unconditional one by changing the order of integration with respect to Lebesgue measure and with respect to the distribution of $X_{m+1}, \ldots, X_{N}$, for $|t| \leqslant C N^{1 / 2}$. Finally, by means of expansions we estimate the difference between the Fourier-Stieltjes transforms of $F$ and $G$.

Our proofs may be considered as an extension to the case of finite-population statistics of techniques used by Bentkus et al. (1997) in the i.i.d. case. We remark that the approach developed in the present paper also applies to more general nonlinear symmetric statistics based on samples drawn without replacement from finite populations. These results will appear elsewhere.

\subsection{Notation}

By $C, C_{0}, C_{1}, \ldots$ and $c, c_{0}, c_{1}, \ldots$ we denote generic absolute constants. We shall write $A \ll B$ if $A<C B$. The expression $\exp \{\mathrm{i} x\}$ will be abbreviated to $\{x\}$. Write

$$
\Theta(t)=\left(\frac{2}{\pi} \frac{\pi-t}{\pi+t}\right)^{2}, \quad \mathscr{K}=\left\{a \in \mathscr{b}: H_{1}\left|g_{1}(a)\right|<b_{2}\right\}, \quad H_{1}=\frac{b_{1} N^{1 / 2}}{\beta_{3}} .
$$

Here $b_{1}$ is the same constant as in (1.5) and $b_{2}$ denotes a sufficiently small absolute constant.

Let $v=\left\{v_{1}, \ldots, v_{n}\right\}$ be a sequence of independent Bernoulli random variables with probabilities $\mathrm{P}\left\{v_{i}=1\right\}=p$ and $\mathrm{P}\left\{v_{i}=0\right\}=q$, for $i=1,2, \ldots, n$. Write

$$
\beta(t)=\operatorname{Ee}\left\{\left(v_{1}-p\right) t\right\}, \quad \tau=\sqrt{n p q}, \quad \delta=\delta\left(b_{1} / \beta_{3}, N^{1 / 2}\right),
$$

where $\delta(\cdot, \cdot)$ is defined by $(2.1)$. Let $\bar{A}=\left(A_{1}, A_{2}, \ldots, A_{n}\right)$ denote a random permutation which is uniformly distributed on the permutations of the ordered set $\left(a_{1}, \ldots, a_{n}\right)$ of elements of $\mathscr{A}$, independent of $\nu$. By $\mathrm{E}^{*}$ we denote the conditional expectation given $\bar{A}$, i.e. $\mathrm{E}^{*}(\cdot)=\mathrm{E}(\cdot \mid \bar{A})$. Fo $k=1,2, \ldots, \quad$ write $\quad \Omega_{k}=\{1, \ldots, k\} \quad$ and $\quad D_{k}=\Omega_{N} \backslash \boldsymbol{\Omega}_{k}$. Given 
$D=\left\{i_{1}, i_{2}, \ldots, i_{k}\right\} \subset \Omega_{n}, \mathrm{E}^{i_{1}, \ldots, i_{k}}$ and $\mathrm{E}^{D}$ denote the conditional expectation given $A_{i_{1}}, \ldots, A_{i_{k}}$.

\subsection{Proof of Theorem 1.1}

We may and shall assume that, for sufficiently small $c_{0}>0$,

$$
\frac{\beta_{4}}{q N}<c_{0}, \quad \frac{\ln N}{\delta N}<c_{0}, \quad \frac{\gamma_{2}}{\delta^{2} q^{2} N}<c_{0}, \quad \frac{\ln N}{\delta q n}<c_{0} .
$$

Indeed, if (3.3) fails, then the bound of Theorem 1.1 follows from the inequalities $F(x) \leqslant 1$, $|G(x)| \ll 1+q^{-1 / 2} \beta_{4}^{1 / 2} / N^{1 / 2}+q^{1 / 2} \gamma_{2} / N^{1 / 2}$ and $\rho \leqslant \delta$; see Lemma 2.1 .

Step 1. Fix an integer $m \approx C_{0} \delta^{-1} \ln N$, with sufficiently large $C_{0}$, and write

$$
\Lambda_{m}=\sum_{1 \leqslant k<l \leqslant m} g_{2}\left(X_{k}, X_{l}\right) .
$$

Note that $U=\Lambda_{m}+U_{1}$, where $U_{1}$ is given by (3.1). Let $F_{1}$ denote the probability distribution function of $U_{1}$ and $\Delta_{1}=\sup _{x}\left|F_{1}(x)-G(x)\right|$. We have

$$
\Delta \leqslant \Delta_{1}+\mathrm{P}\left\{\left|\Lambda_{m}\right| \geqslant N^{-1} \delta^{-3 / 2}\right\}+\delta^{-3 / 2} N^{-1} \max _{x}\left|G^{\prime}(x)\right| .
$$

By Chebyshev's inequality and the inequality $\mathrm{E}\left|\Lambda_{m}\right|^{3} \ll m^{6} \mathrm{E}\left|g_{2}\left(X_{1}, X_{2}\right)\right|^{3}$,

$$
\mathrm{P}\left\{\left|\Lambda_{m}\right| \geqslant N^{-1} \delta^{-3 / 2}\right\} \leqslant \delta^{9 / 2} N^{3} \mathrm{E}\left|\Lambda_{m}\right|^{3} \ll \delta^{-3 / 2} \gamma_{3} N^{-3 / 2} \ln ^{6} N .
$$

Finally, using the bound $\left|G^{\prime}(x)\right| \ll \beta_{4} / q+\gamma_{2}$ we obtain

$$
\Delta \ll \Delta_{1}+N^{-1} \delta^{-3 / 2}\left(\beta_{4} / q+\gamma_{2}+\gamma_{3}\right) .
$$

Therefore, in order to prove the theorem it suffices to bound $\Delta_{1}$.

Let $k$ be an integer approximately equal to $(N+m) / 2$. Put $\mathscr{T}_{0}=\{m+1, \ldots, N\}$, $\mathscr{J}_{0}=\Omega_{n} \backslash \mathscr{T}_{0}, \mathscr{J}_{1}=\mathscr{J}_{0} \cup\{m+1, \ldots, k\}$ and $\mathscr{J}_{2}=\mathscr{J}_{0} \cup\{k+1, \ldots, N\}$. Given $\bar{A}$, define (random) subpopulations $\mathscr{C}_{i}=\left\{A_{k}, k \in \mathscr{J}_{i}\right\}, i=0,1,2$, and let $A_{i}^{*}$ be random variables uniformly distributed in $\mathscr{A}_{i}, i=0,1,2$, indpendent of $v$. Write

$$
\begin{gathered}
v_{1}(a)=\sum_{j=k+1}^{N} g_{2}\left(a, A_{j}\right), \quad v_{2}(a)=\sum_{j=m+1}^{k} g_{2}\left(a, A_{j}\right), \\
H=N \delta /\left(32 q^{-1} N\left(\Theta_{1}+\Theta_{2}\right)+1\right), \quad \Theta_{i}=\mathrm{E}^{*}\left|v_{i}\left(A_{i}^{*}\right)\right|, \quad i=1,2 .
\end{gathered}
$$

Notice that $\Theta_{1}$ is a function of the random variables $A_{k+1}, \ldots, A_{N}$, and that $\Theta_{2}$ is a function of $A_{m+1}, \ldots, A_{k}$.

Step 2. Split the sample as follows. Put $X_{j}=A_{j}$, for $m<j \leqslant N$. The rest of the sample, $X_{1}, \ldots, X_{m}$, is obtained by simple random sampling without replacement from the (random) subpopulation $\mathscr{b}_{0}$.

An application of Prawitz's (1972) smoothing lemma conditionally, given $X_{m+1}, \ldots, X_{m}$, or equivalently, given $A_{m+1}, \ldots, A_{N}$, gives 


$$
\begin{aligned}
& F_{1}(x+) \leqslant \frac{1}{2}+\operatorname{EVP} \int_{\mathbb{R}} \mathrm{e}\{-x t\} \frac{1}{H} K\left(\frac{t}{H}\right) f_{1}(t) \mathrm{d} t \\
& F_{1}(x-) \geqslant \frac{1}{2}-\operatorname{EVP} \int_{\mathbb{R}} \mathrm{e}\{-x t\} \frac{1}{H} K\left(\frac{-t}{H}\right) f_{1}(t) \mathrm{d} t
\end{aligned}
$$

where $2 K(s)=K_{1}(s)+\mathrm{i} K_{2}(s) /(\pi s)$; see, for example, Bentkus et al. (1997). Here

$$
K_{1}(s)=\mathrm{I}\{|s| \leqslant 1\}(1-|s|) \quad \text { and } \quad K_{2}(s)=\mathrm{I}\{|s| \leqslant 1\}(1-|s|) \pi s \cot (\pi s) .
$$

Combining (3.7) and the inversion formula,

$$
G(x)=\frac{1}{2}+\frac{\mathrm{i}}{2 \pi} \lim _{M \rightarrow \infty} \mathrm{VP} \int_{|t| \leqslant M} \mathrm{e}\{-t x\} \hat{G}(t) \frac{\mathrm{d} t}{t},
$$

we obtain (see, for example, Bentkus et al. 1997)

$$
\begin{aligned}
& F_{1}(x+)-G(x) \leqslant \mathrm{E} I_{1}+\mathrm{E} I_{2}+\mathrm{E} I_{3}, \\
& I_{1}=\frac{1}{2} H^{-1} \int_{\mathbb{R}} \mathrm{e}\{-x t\} K_{1}\left(\frac{t}{H}\right) f_{1}(t) \mathrm{d} t, \\
& I_{2}=\frac{\mathrm{i}}{2 \pi} \mathrm{VP} \int_{\mathbb{R}} \mathrm{e}\{-x t\} K_{2}\left(\frac{t}{H}\right)\left(f_{1}(t)-\hat{G}(t)\right) \frac{\mathrm{d} t}{t}, \\
& I_{3}=\frac{\mathrm{i}}{2 \pi} \operatorname{VP} \int_{\mathbb{R}} \mathrm{e}\{-x t\}\left(K_{2}\left(\frac{t}{H}\right)-1\right) \tilde{G}(t) \frac{\mathrm{d} t}{t},
\end{aligned}
$$

where VP means also that one should take $\lim _{M \rightarrow \infty}$ if necessary.

Combining (3.8) and (3.9), we obtain a bound for $G(x)-F_{1}(-x)$ similar to (3.10). We shall bound $F_{1}(x+)-G(x)$ only. To this end, we prove that

$$
\left|\mathrm{E} I_{1}\right|+\left|\mathrm{E}\left(I_{2}+I_{3}\right)\right| \ll N^{-1}\left(\beta_{4} / q+\delta^{-1}\left(\delta^{-1}+q^{-1}\right)+\delta^{-2} q^{-2}\left(\gamma_{2}^{1 / 2}+\gamma_{2}\right)+\gamma_{4}\right) .
$$

The analogous bound for $G(x)-F_{1}(x-)$ can be derived in the same way. Using these bounds, (3.5) and the inequality $\delta \geqslant \rho$ (see Lemma 2.1), we obtain the estimate of the theorem. in the remaining part of the prooof we verify (3.11).

Step 3: Estimate for $\left|\mathrm{E} I_{1}\right|$. We shall replace the random bound $H$ in the integral $I_{1}$ by a non-random one and $K_{1}(t / H)$ by 1 . We have $\left|\mathrm{E} I_{1}\right| \leqslant\left|\mathrm{E} I_{4}\right|+\mathrm{E} I_{5}$, where

$$
\begin{aligned}
& I_{4}=H^{-1} \int_{\mathscr{L}} \mathrm{e}\{-t x\} K_{1}\left(\frac{t}{H}\right) f_{1}(t) \mathrm{d} t, \quad \mathscr{Z}=\left\{t \in \mathbb{R}:|t| \leqslant H_{1}\right\}, \\
& I_{5}=H^{-1} \int_{H_{1} \leqslant|t|} K_{1}\left(\frac{t}{H}\right)\left|f_{1}(t)\right| \mathrm{d} t \leqslant H^{-1} \int_{H_{1} \leqslant|t| \leqslant H}\left|f_{1}(t)\right| \mathrm{d} t .
\end{aligned}
$$

Next we construct bounds for $\mathrm{E} I_{5}$ and $\left|\mathrm{E} I_{4}\right|$; see (3.12) and (3.19) below. It follows from these bounds that $\left|\mathrm{E} I_{1}\right|$ does not exceed the right-hand side of (3.11).

Let us show 


$$
\mathrm{E} I_{5} \ll N^{-2} \beta_{3} .
$$

For this purpose we represent $f_{1}(t)$ in Erdös and Rényi (1959) form conditionally, given $A_{m+1}, \ldots, A_{N}$. Let $v^{*}=\left\{v_{1}^{*}, \ldots, v_{n}^{*}\right\}$ be a sequence of independent Bernoulli random variables independent of $\overline{\mathscr{C}}$ and with probabilities

$$
\mathrm{P}\left\{v_{i}^{*}=1\right\}=p^{*}, \quad \mathrm{P}\left\{v_{i}^{*}=0\right\}=q^{*}, \quad p^{*}=\frac{m}{n-(N-m)}, \quad q^{*}=1-p^{*} .
$$

Write $S_{*}=\sum_{k \in \mathscr{Z}_{0}}\left(v_{k}^{*}-p^{*}\right)$ and $L_{*}=\sum_{k \in \mathscr{Z}_{0}} l\left(A_{k}\right) v_{k}^{*}$. We have

$$
f_{1}(t)=\mathrm{P}^{-1}\left\{S_{*}=0\right\} \frac{1}{2 \pi} \int_{-\pi}^{\pi} W \mathrm{~d} s, \quad W=\mathrm{E}^{*} \mathrm{e}\left\{t\left(L_{*}+U^{\prime}\right)+s S_{*}\right\} .
$$

We shall construct an upper bound for $|W|$. We have

$$
|W|=\prod_{k \in \mathscr{Z}_{0}}\left|\beta_{*}\left(z\left(A_{k}\right)+t v\left(A_{k}\right)\right)\right|, \quad \beta_{*}(x)=\operatorname{Ee}\left\{\left(v_{1}^{*}-p^{*}\right) x\right\} .
$$

Here we denote

$$
z(a)=\operatorname{tg}_{1}(a)+s \quad \text { and } \quad v(a)=v_{1}(a)+v_{2}(a),
$$

with $v_{i}(a)$ given by (3.6). Then we apply the identity $\left|\beta_{*}(x)\right|^{2}=1-2 p^{*} q^{*}(1-\cos x)$ to $x=z(a)+t v(a)$ and expand the cosine function in powers of $t v(a)$ to obtain

$$
\begin{gathered}
\left|\beta_{*}(z(a)+t v(a))\right|^{2} \leqslant u_{1}(a)+u_{2}(a), \\
u_{1}(a)=1-2 p^{*} q^{*}(1-\cos (z(a))), \quad u_{2}(a)=2 p^{*} q^{*}|t v(a)| .
\end{gathered}
$$

Furthermore, we may assume that $p^{*} \leqslant 8^{-1}$ (this is a consequence of the last inequality of (3.3) provided that $c_{0}$ is small enough). This inequality implies $u_{1}(a) \geqslant 1 / 2$ and, therefore,

$$
u_{1}(a)+u_{2}(a) \leqslant u_{1}(a)\left(1+2 u_{2}(a)\right) .
$$

Combining (3.14) and (3.15), we obtain

$$
|W|^{2} \leqslant W_{1} W_{2}, \quad W_{1}=\prod_{k \in \mathscr{Z}_{0}} u_{1}\left(A_{k}\right), \quad W_{2}=\prod_{k \in \mathscr{Z}_{0}}\left(1+2 u_{2}\left(A_{k}\right)\right) .
$$

To estimate $W_{2}$, we apply the arithmetic-geometric mean inequality,

$$
W_{2} \leqslant\left(\frac{1}{\left|\mathscr{J}_{0}\right|} \sum_{k \in \mathscr{Z}_{0}}\left(1+2 u_{2}\left(A_{k}\right)\right)\right)^{\left|\mathscr{Z}_{0}\right|}=\left(\mathrm{E}^{*}\left(1+2 u_{2}\left(A_{0}^{*}\right)\right)\right)^{n-N+m},
$$

and use (5.2) to bound $\mathrm{E}^{*}\left|v\left(A_{0}^{*}\right)\right| \leqslant q^{-1}\left(\Theta_{1}+\Theta_{2}\right)$. Thus, for $|t| \leqslant H$, we obtain

$$
\mathrm{E}^{*}\left(1+2 u_{2}\left(A_{0}^{*}\right)\right) \leqslant 1+4 p^{*} q^{*} q^{-1} H\left(\Theta_{1}+\Theta_{2}\right) \leqslant 1+p^{*} q^{*} \frac{\delta}{8} \leqslant \exp \left\{p^{*} q^{*} \frac{\delta}{8}\right\} .
$$

This inequality, in combination with (3.17), implies $W_{2}^{1 / 2} \leqslant \exp \left\{m q^{*} \delta / 16\right\}$. Now in view of (3.16) and (3.13) we obtain, for $|t| \leqslant H$,

$$
\left|f_{1}(t)\right| \ll W_{3} W_{1}^{1 / 2}, \quad W_{3}=m^{1 / 2} \exp \left\{m q^{*} \delta / 16\right\} .
$$


Here we have estimated $P^{-1}\left\{S_{*}=0\right\} \ll m^{1 / 2}$; see (5.16). We have

$$
\mathrm{E} I_{5} \leqslant \frac{1}{H_{1}} \mathrm{E} \int_{H_{1} \leqslant|t| \leqslant H}\left|f_{1}(t)\right| \mathrm{d} t \leqslant \frac{W_{3}}{H_{1}} \int_{H_{1} \leqslant|t| \leqslant N} \mathrm{E} W_{1}^{1 / 2} \mathrm{~d} t .
$$

To bound $\mathrm{E} W_{1}^{1 / 2}$ we apply Hölder's inequality and Theorem 4 of Hoeffding (1963),

$$
\left(\mathrm{E} W_{1}^{1 / 2}\right)^{2} \leqslant \mathrm{E} W_{1} \leqslant\left(\mathrm{E} u_{1}\left(A_{1}\right)\right)^{\left|\mathscr{Z}_{0}\right|} ;
$$

see Section 5 below. Note that $\mathrm{E} u_{1}\left(A_{1}\right) \leqslant 1-2 p^{*} q^{*} \delta$, for $H_{1} \leqslant|t| \leqslant N$, by the choice of $\delta$. Therefore,

$$
\mathrm{E} W_{1}^{1 / 2} \leqslant\left(1-2 p^{*} q^{*} \delta\right)^{(n-N+m) / 2} \leqslant \exp \left\{-p^{*} q^{*} \delta(n-N+m)\right\}=\exp \left\{-m q^{*} \delta\right\} .
$$

Combining this bound with (3.18) and using the inequality $q^{*}=1-p^{*} \geqslant 7 / 8$, we obtain (3.12), provided that the constant $C_{0}$ (in the definition of $m$ ) is sufficiently large.

We must now bound $\mathrm{E}_{4}$. We shall show

$$
\left|\mathrm{E} I_{4}\right| \ll \mathscr{R}_{0}, \quad \mathscr{R}_{0}=N^{-1} \delta^{-2}\left(1+q^{-2} \gamma_{2}\right)+N^{-1} \delta^{-1} q^{-1}\left(1+q^{-1} \gamma_{2}^{1 / 2}\right) .
$$

It follows from the inequality $\left|K_{1}(u)-1\right| \leqslant|u|$ that

$$
\begin{aligned}
& I_{4}=I_{6}+R, \quad I_{6}=H^{-1} \int_{\mathscr{L}} \mathrm{e}\{-t x\} f_{1}(t) \mathrm{d} t, \\
& \mathrm{E}|R| \leqslant \mathrm{E} H^{-1} \int_{\mathscr{L}}|t| H^{-1} \mathrm{~d} t=H_{1}^{2} \mathrm{E} H^{-2} \ll \mathscr{R}_{0},
\end{aligned}
$$

where in the last step we have applied (5.1). Recall that $U=U_{1}+\Lambda_{m}$. Now, using the inequality $\left|\mathrm{e}\left\{t \Lambda_{m}\right\}-1\right| \leqslant\left|t \Lambda_{m}\right|$, we obtain

$$
\begin{gathered}
I_{6}=I_{7}+R, \quad I_{7}=H^{-1} \int_{\mathscr{E}} \mathrm{e}\{-t x\} f_{2}(t) \mathrm{d} t, \quad f_{2}(t)=\mathrm{E}^{D_{m}} \mathrm{e}\{t U\}, \\
\mathrm{E}|R| \leqslant \mathrm{E} H^{-1} \int_{\mathscr{E}} \mathrm{E}^{D_{m}}\left|t \Lambda_{m}\right| \mathrm{d} t \leqslant H_{1}^{2} \mathrm{E} H^{-1}\left|\Lambda_{m}\right| \ll \mathscr{B}_{0},
\end{gathered}
$$

where in the last step we have used the inequality $\left|\Lambda_{m} H^{-1}\right| \leqslant \Lambda_{m}^{2}+H^{-2}$ and moment inequalities (5.1) and (5.3). Next we replace $I_{7}$ by

$$
I_{8}=H^{-1} \int_{\mathscr{\mathscr { D }}_{0}} \mathrm{e}\{-t x\} f_{2}(t) \mathrm{d} t, \quad \mathscr{Z}_{0}=\left\{C_{1} q^{-1} \leqslant|t| \leqslant H_{1}\right\},
$$

where $C_{1}$ is a sufficiently large constant. We have $I_{7}=I_{8}+R$ with $|R| \leqslant 2 C_{1} q^{-1} H^{-1}$. Hölder's inequality, in combination with (5.1), gives $\mathrm{E}|R| \ll \mathscr{B}_{0}$.

It remains to estimate $\mathrm{E} I_{8}$. Write $I_{8}=32 q^{-1} \delta^{-1}\left(J_{1}+J_{2}\right)+\delta^{-1} J_{3}$, where

$$
J_{i}=\int_{\mathscr{D}_{0}} \mathrm{e}\{-t x\} f_{2}(t) \Theta_{i} \mathrm{~d} t, \quad i=1,2, \quad J_{3}=N^{-1} \int_{\mathscr{D}_{0}} \mathrm{e}\{-t x\} f_{2}(t) \mathrm{d} t .
$$

In order to complete the prooof of (3.19), we shall show

$$
\text { E } J_{i} \ll N^{-1}\left(1+\gamma_{2}\right), \quad i=1,2,3 .
$$


Let us prove (3.23) for $i=1,2$. By symmetry, it suffices to consider the case where $i=1$. Recall that the random variable $\Theta_{1}$ is a function of $X_{k+1}, \ldots, X_{N}$. In view of the inequality $k \approx(N+m) / 2>m$, we can write

$$
\mathrm{E} f_{2}(t)=\mathrm{E} f_{1} f_{3}(t), \quad f_{3}(t)=\mathrm{E}\left(\mathrm{e}\{t U\} \mid X_{k+1}, \ldots, X_{N}\right) .
$$

Given $t \in \mathscr{Z}_{0}$, choose an integer $m_{1}=C_{2} N t^{-2} \ln |t|$. Here $C_{2}$ is a sufficiently large constant to be specified later. Given $C_{2}$, we may choose $C_{1}$ in (3.22) large enough so that $m_{1}<10^{-1} q N<k$, for $t \in \mathscr{E}_{0}$. Write $\mathscr{J}_{3}=\Omega_{m_{1}} \cap\left(\Omega_{n} \backslash \Omega_{N}\right)$. We shall represent our sample $X_{1}, \ldots, X_{N}$ as follows. For $m_{1}+1 \leqslant j \leqslant N$, put $X_{j}=A_{j}$. The remaining part of the sample (the observations $X_{1}, \ldots, X_{m_{1}}$ ) represents a simple random sample drawn without replacement form the set $\mathscr{C}_{3}=\left\{A_{k}, k \in \mathscr{J}_{3}\right\}$. Let $A_{3}^{*}$ be a random variable uniformly distributed in $\mathscr{C b}_{3}$. Put

$$
v_{3}(a)=\sum_{k=m_{1}+1}^{N} g_{2}\left(a, A_{j}\right) \quad \text { and } \quad \Theta_{3}=\mathrm{E}^{*}\left|v_{3}\left(A_{3}^{*}\right)\right| .
$$

Notice that the random variable $\Theta_{3}$ is a function of $A_{m_{1}+1}, \ldots, A_{N}$.

Write $U=U_{1}^{\star}+\Lambda_{m_{1}}$, where $U_{1}^{\star}=L_{\star}^{\prime}+U_{\star}^{\prime}$, with

$$
L_{\star}^{\prime}=l_{\star}\left(X_{1}\right)+\ldots+l_{\star}\left(X_{m_{1}}\right), \quad l_{\star}(x)=g_{1}(x)+l_{0}^{\star}(x), \quad l_{0}^{\star}(x)=\sum_{j=m_{1}+1}^{N} g_{2}\left(x, X_{j}\right),
$$

and with $U_{\star}^{\prime}$ defined by (3.1), but with $m$ replaced by $m_{1}$. Furthermore, $\Lambda_{m_{1}}$ is given by (3.4).

Using the inequality $\left|\mathrm{e}\left\{t \Lambda_{m_{1}}\right\}-1\right| \leqslant\left|t \Lambda_{m_{1}}\right|$, we obtain

$$
\mathrm{E} \Theta_{1} f_{3}(t)=\mathrm{E} \Theta_{1} f_{4}(t)+R_{1}, \quad f_{4}(t)=\mathrm{E}\left(\mathrm{e}\left\{t U_{1}^{*}\right\} \mid X_{k+1}, \ldots, X_{N}\right),
$$

where $\left|R_{1}\right| \leqslant \mathrm{E}\left|t \Lambda_{m_{1}}\right| \Theta_{1}$. Furthermore, combining (3.24) and (3.26), we obtain

$$
\begin{gathered}
\mathrm{E} J_{1}=\mathrm{E} J_{4}+R, \quad J_{4}=\int_{\mathscr{D}_{0}} \mathrm{e}\{-t x\} f_{4}(t) \Theta_{1} \mathrm{~d} t, \\
|R| \leqslant \int_{\mathscr{D}_{0}} \mathrm{E}\left|t \Lambda_{m_{1}}\right| \Theta_{1} \mathrm{~d} t \ll N^{-1} \gamma_{2} .
\end{gathered}
$$

In the last step we invoke (5.1), (5.3) and apply Hölder's inequality to obtain

$$
\mathrm{E}\left|\Lambda_{m_{1}}\right| \Theta_{1} \leqslant\left(\mathrm{E}_{m_{1}}^{2}\right)^{1 / 2}\left(\mathrm{E} \Theta_{1}^{2}\right)^{1 / 2} \ll m_{1} N^{-5 / 2} \gamma_{2} \ll t^{-1} \ln |t| N^{-3 / 2} \gamma_{2}
$$

and bound the integral of the function $|t|^{-1} \ln |t|$ over the region $\mathscr{Z}_{0}$ by $\ln ^{2} N$.

To estimate $\mathrm{E} J_{4}$ observe that, by the inequality $m_{1}<k$,

$$
\mathrm{E}_{1} f_{4}=\mathrm{E}_{1} f_{5}, \quad f_{5}=\mathrm{E}\left(\mathrm{e}\left\{t U_{\star}^{\prime}\right\} \mid X_{m_{1}+1}, \ldots, X_{N}\right) .
$$

Therefore, $\mathrm{E} J_{4}=\mathrm{E} J_{5}$, where $J_{5}$ is defined in the same way as $J_{4}$ (see (3.27)) but with $f_{4}$ replaced by $f_{5}$. Furthermore,

$$
\mathrm{E} J_{5}=\mathrm{E} J_{6}+R, \quad J_{6}=\int_{\mathscr{\infty}_{0}} \mathrm{e}\{-t x\} f_{5}(t) \Theta_{1} I_{\Theta} \mathrm{d} t
$$




$$
I_{\Theta}=I\left\{N \Theta_{3} \leqslant c_{1}|t|\right\}, \quad|R| \leqslant \int_{\mathscr{D}_{0}} \mathrm{E}_{1} I\left\{N \Theta_{3}>c_{1}|t|\right\} \mathrm{d} t \ll N \Theta_{1} \Theta_{3} .
$$

Here $c_{1}$ denotes a small positive constant to be determined below. Combining (5.3) and Hölder's inequality, we obtain $|R| \ll N^{-1} \gamma_{2}$.

In order to bound $\mathrm{E} J_{6}$ we represent $f_{5}$ in the Erdös and Rényi (1959) form; see (3.29). Let $v^{\star}=\left\{v_{1}^{\star}, \ldots, v_{n}^{\star}\right\}$ be a sequence of independent Bernoulli random variables independent of $\overline{\mathscr{b}}$ and with probabilities

$$
\mathrm{P}\left\{v_{i}^{\star}=1\right\}=p^{\star}, \quad \mathrm{P}\left\{v_{i}^{\star}=0\right\}=q^{\star}, \quad p^{\star}=\frac{m_{1}}{n-\left(N-m_{1}\right)}, \quad q^{\star}=1-p^{\star} .
$$

Write $S_{\star}=\sum_{k \in \mathscr{Z}_{3}}\left(v_{k}^{\star}-p^{\star}\right), L_{\star}=\sum_{k \in \mathscr{Z}_{3}} l_{\star}\left(A_{k}\right) v_{k}^{\star}$ and $\tau_{\star}^{2}=m_{1} q^{\star}$. We have

$$
f_{5}(t)=\lambda_{\star} \int_{-\pi \tau_{\star}}^{\pi \tau_{\star}} W_{\star} \mathrm{d} s, \quad W_{\star}=\mathrm{E}^{*} \mathrm{e}\left\{t\left(L_{\star}+U_{\star}^{\prime}\right)+\frac{s}{\tau_{\star}} S_{\star}\right\},
$$

with $\lambda_{\star}^{-1}=2 \pi \tau_{\star} \mathrm{P}\left\{S_{\star}=0\right\}$ satisfying $\lambda_{\star} \ll 1$, by (5.16).

Combining (3.28) and (3.29), we obtain

$$
\mathrm{E} J_{6} \ll \int_{\mathscr{\otimes}_{0}} \mathrm{~d} t \int_{-\pi \tau_{\star}}^{\pi \tau_{\star}} \mathrm{E} \Theta_{1} I_{\Theta}\left|W_{\star}\right| \mathrm{d} s .
$$

In the next step we construct an upper bound for $\mathrm{E} \Theta_{1} I_{\Theta}\left|W_{\star}\right|$. Note that the inequality $m_{1}<10^{-1} q N$ implies $p^{\star} \leqslant 10^{-1}$. The same argument as above (see (3.16)) gives

$$
\left|W_{\star}\right|^{2} \leqslant W_{1}^{\star} W_{2}^{\star}, \quad W_{1}^{\star}=\prod_{k \in \mathscr{Z}_{3}} u_{1}^{\star}\left(A_{k}\right), \quad W_{2}^{\star}=\prod_{k \in \mathscr{Z}_{3}}\left(1+2 u_{2}^{\star}\left(A_{k}\right)\right),
$$

where $u_{1}^{\star}$ and $u_{2}^{\star}$ are given by (3.14), but with $p^{*}, q^{*}, z(a)$ and $v(a)$ replaced by $p^{\star}, q^{\star}$, $z_{\star}(a):=\operatorname{tg}_{1}(a)+s / \tau_{\star}$ and $v_{3}(a)$ (defined in (3.25)) respectively.

To bound $W_{2}^{\star}$ we proceed as in (3.17) and obtain

$$
W_{2}^{\star} \leqslant\left(1+2 \mathrm{E}^{*} u_{2}^{\star}\left(A_{3}^{*}\right)\right)^{n-N+m_{1}}=\left(1+4 p^{\star} q^{\star}|t| \Theta_{3}\right)^{n-N+m_{1}} \leqslant \exp \left\{4 m_{1} q^{\star}|t| \Theta_{3}\right\} .
$$

Furthermore, by our choice of $m_{1}, I_{\Theta}\left(W_{2}^{\star}\right)^{1 / 2} \leqslant \exp \left\{2 q^{\star} C_{2} c_{1} \ln |t|\right\}$. Therefore, in view of (3.31),

$$
\mathrm{E}_{1} I_{\Theta}\left|W_{\star}\right| \leqslant \exp \left\{2 q^{\star} C_{2} c_{1} \ln |t|\right\} \mathrm{E}_{1}\left(W_{1}^{\star}\right)^{1 / 2} .
$$

Now we apply Hölder's inequality and invoke (5.1) to obtain

$$
\mathrm{E} \Theta_{1}\left(W_{1}^{\star}\right)^{1 / 2} \leqslant\left(\mathrm{E} \Theta_{1}^{2}\right)^{1 / 2}\left(\mathrm{E} W_{1}^{\star}\right)^{1 / 2} \ll N^{-1} \gamma_{2}^{1 / 2}\left(\mathrm{E} W_{1}^{\star}\right)^{1 / 2} .
$$

To bound $\mathrm{E} W_{1}^{\star}$ we apply Theorem 4 of Hoeffding (1963) and obtain

$$
\mathrm{E} W_{1}^{\star} \leqslant\left(\mathrm{E} u_{1}^{\star}\left(A_{1}\right)\right)^{\left|\mathscr{Z}_{3}\right|}=\left(1-2 p^{\star} q^{\star} M\right)^{n-N+m_{1}} \leqslant \exp \left\{-2 m_{1} q^{\star} M\right\},
$$

where $M=\mathrm{E}\left(1-\cos z_{\star}\left(A_{1}\right)\right)$. Combining the inequalities

$$
\begin{gathered}
M \geqslant \mathrm{E}\left(1-\cos z_{\star}\left(A_{1}\right)\right) I_{\mathscr{K}}\left(A_{1}\right), \quad I_{\mathscr{K}}(a)=I\{a \in \mathscr{K}\}, \\
1-\cos z_{\star}(a) \geqslant 2^{-1} \Theta\left(b_{2}\right) z_{\star}^{2}(a), \quad a \in \mathscr{K},
\end{gathered}
$$


(see (5.15)) we get $M \geqslant 2^{-1} \Theta\left(b_{2}\right) \mathrm{E} z_{\star}^{2}\left(A_{1}\right) I_{\mathscr{K}}\left(A_{1}\right)$. Now, by Lemma 5.3,

$$
M \geqslant b_{3}\left(t^{2} N^{-1}+s^{2} \tau_{\star}^{-2}\right), \quad b_{3}=2^{-1} \Theta\left(b_{2}\right)\left(1-2 b_{1} b_{2}^{-1}\right),
$$

is a positive constant (because of our choice of $0<2 b_{1}<b_{2}$ in (1.5) and (3.2)). Substituting this inequality in (3.34) and using $q^{\star}=1-p^{\star} \geqslant 9 / 10$, we obtain

$$
\mathrm{E} W_{1}^{\star} \leqslant \exp \left\{-2 b_{3} m_{1} q^{\star}\left(t^{2} N^{-1}+s^{2} \tau_{\star}^{-2}\right)\right\} \leqslant \exp \left\{-2 b_{3}\left(\frac{9}{10} C_{2} \ln |t|+s^{2}\right)\right\} .
$$

Finally, collecting the inequalities (3.32), (3.33) and (3.35) in (3.30), we obtain

$$
\mathrm{E} J_{6} \ll N^{-1} \gamma_{2}^{1 / 2} \int_{\mathscr{D}_{0}} \mathrm{~d} t \int_{-\pi \tau_{\star}}^{\pi \tau_{\star}} \exp \left\{C_{2}\left(2 c_{1}-\frac{9}{10} b_{3}\right) \ln |t|-b_{3} s^{2}\right\} \mathrm{d} s .
$$

Choosing $c_{1}=b_{3} / 4$ and $C_{2}=4 / b_{3}$, we obtain bounded integrals in (3.36), and thus E $J_{6} \ll N^{-1} \gamma_{2}^{1 / 2} \leqslant N^{-1}\left(1+\gamma_{2}\right)$. This inequality, together with (3.27) and (3.28), completes the proof of (3.23) in the case where $i=1$.

The proof of (3.23) in the case where $i=3$ is similar but simpler: just write $N^{-1}$ instead of $\Theta_{1}$ in the proof above.

Collecting the bounds (3.20), (3.21), (3.22) and (3.23), we obtain (3.19).

Step 4. Estimate for $\left|\mathrm{E}\left(I_{2}+I_{3}\right)\right|$. Write $I_{2}+I_{3}=\mathrm{i}(2 \pi)^{-1}\left(I_{9}+I_{10}-I_{11}+I_{12}\right)$, where

$$
\begin{aligned}
I_{9} & =\int_{|t| \leqslant H_{1}} \mathrm{e}\{-t x\} \frac{f_{1}(t)-\hat{G}(t)}{t} \mathrm{~d} t, I_{10}=\int_{H_{1} \leqslant|t| \leqslant H} \mathrm{e}\{-t x\} K_{2}\left(\frac{t}{H}\right) f_{1}(t) \frac{\mathrm{d} t}{t}, \\
I_{11} & =\int_{|t|>H_{1}} \mathrm{e}\{-t x\} \hat{G}(t) \frac{\mathrm{d} t}{t}, I_{12}=\int_{|t| \leqslant H_{1}} \mathrm{e}\{-t x\}\left(K_{2}\left(\frac{t}{H}\right)-1\right) f_{1}(t) \frac{\mathrm{d} t}{t} .
\end{aligned}
$$

Using (3.3), it is easy to show that $\left|\mathrm{E} I_{11}\right| \ll q^{-1} \beta_{4} / N+\gamma_{2} / N$. Using the inequality $\left|K_{2}(s)-1\right| \leqslant c s^{2}$, and invoking (5.1), we obtain

$$
\left|\mathrm{E} I_{12}\right| \ll \mathrm{E} H^{-2} H_{1}^{2} \ll \delta^{-2} N^{-1}\left(1+q^{-2} \gamma_{2}\right) .
$$

To bound $\left|\mathrm{E} I_{10}\right|$ write

$$
\left|\mathrm{E} I_{10}\right| \leqslant \mathrm{E} I_{13}, I_{13}=\int_{H_{1} \leqslant|t| \leqslant H}\left|f_{1}(t)\right| \frac{\mathrm{d} t}{|t|} .
$$

The bound $\mathrm{E} I_{13} \ll N^{-1} \beta_{3}$ is obtained in a similar way as (3.12) above. Collecting these inequalities, we obtain

$$
\left|\mathrm{E}\left(I_{2}+I_{3}\right)\right| \ll\left|\mathrm{E} I_{9}\right|+N^{-1} q^{-1} \beta_{4}+N^{-1} \delta^{-2}\left(1+q^{-2} \gamma_{2}\right) .
$$

In order to complete the proof of (3.11) we shall show that

$$
\left|\mathrm{E} I_{9}\right| \ll \delta^{-2} N^{-1}\left(1+\gamma_{2}\right)+N^{-1}\left(q^{-1} \beta_{4}+\gamma_{4}\right) .
$$

We have

$$
\mathrm{E} I_{9}=\int_{|t| \leqslant H_{1}} \mathrm{e}\{-t x\}\left(\operatorname{Ee}\left\{t U_{1}\right\}-\hat{G}(t)\right) \frac{\mathrm{d} t}{t} .
$$


Recall that $U_{1}=U-\Lambda_{m}$. Write $\mathrm{e}\left\{t U_{1}\right\}=\mathrm{e}\{t U\} \mathrm{e}\left\{-t \Lambda_{m}\right\}$ and expand $\mathrm{e}\left\{-t \Lambda_{m}\right\}$ in powers of $-\mathrm{i} t \Lambda_{m}$ to obtain $\mathrm{E} I_{9}=I_{14}-\mathrm{i} I_{15}+R$, where

$$
I_{14}=\int_{-H_{1}}^{H_{1}} \mathrm{e}\{-t x\} \frac{\hat{F}(t)-\hat{G}(t)}{t} \mathrm{~d} t, I_{15}=\int_{-H_{1}}^{H_{1}} \mathrm{e}\{-t x\} \mathrm{E} \Lambda_{m} \mathrm{e}\{t U\} \mathrm{d} t,
$$

and where $|R| \leqslant H_{1}^{2} \mathrm{E} \Lambda_{m}^{2} \ll \delta^{-2} N^{-1} \gamma_{2}$, by (5.3). By symmetry, $\mathrm{E} I_{15}=\left(\begin{array}{c}m \\ 2\end{array}\right) \mathrm{E} I_{16}$, where $I_{16}$ is defined in the same way as $I_{15}$, but with $\Lambda_{m}$ replaced by $g_{2}\left(X_{N-1}, X_{N}\right)$. the bound $\mathrm{E} I_{16} \ll N^{-3 / 2}\left(1+\gamma_{2}\right)$ is obtained in a similar way to (3.23): just take $\tilde{f}_{3}=$ $\mathrm{E}\left(\mathrm{e}\{t U\} \mid X_{N-1}, X_{N}\right)$ instead of $f_{3}$ and $g_{2}\left(X_{N-1}, X_{N}\right)$ instead of $\Theta_{1}$ in the proof of (3.23) (for $i=1$ ). We obtain

$$
\left|\mathrm{E} I_{9}-I_{14}\right| \ll \delta^{-2} N^{-1}\left(1+\gamma_{2}\right) .
$$

In the next section on expansions (see (4.1) below) we shall show $\left|I_{14}\right| \ll N^{-1}\left(\beta_{4} / q+\gamma_{4}\right)$, thus completing the proof of (3.38).

\subsection{Proof of Theorem 1.2}

The bound of the theorem follows from (3.11). Just note that for a linear statistic we have $g_{2}(x, y)=0$, for any $x, y \in \mathscr{A}$. In particular, we do not need to assume that the last two inequalities of (3.3) hold.

\subsection{Proof of Corollary 1.3}

The corollary follows from Theorem 1.1, by the law of large numbers (LLN) for $U$-statistics; see, for example, Serfling (1980). Given $N$, the function $\mathscr{H}$ and a sequence of i.i.d. observations $\mathscr{B}_{1}, \mathscr{C}_{2}, \ldots$, introduce the sequence of finite populations $\mathscr{C}_{n}=$ $\left\{\mathscr{C}_{1}, \ldots, \mathscr{C}_{n}\right\}$ and the corresponding sequence of $U$-statistics, $\left(U_{n}\right)$. Given $x \in \mathbb{R}$, apply the bound of Theorem 1.1 to the sequence of probabilities $P_{n}\{x\}=\mathrm{P}\left\{U_{n} \leqslant x\right\}$. By the LLN, we obtain $\lim _{n} P_{n}\{x\}=\mathrm{P}\{\tilde{U} \leqslant x\}$. Furthermore, the moments of the linear and quadratic parts of $U_{n}$ in the expansion and in the remainder (in the estimate of Theorem 1.1) converge to the corresponding moments of the statistics $\tilde{U}$, thus proving Corollary 1.3.

\section{Expansions}

As in the previous section, we assume that $\beta_{2}=1$ and that inequalities (3.3) hold. With $H_{1}$ given in (3.2), in this section we shall prove the inequality

$$
\int_{|t| \leqslant H_{1}}|t|^{-1}|\hat{F}(t)-\hat{G}(t)| \mathrm{d} t \ll \mathscr{B}, \quad \mathscr{B}:=\frac{1}{N}\left(\frac{\beta_{4}}{q}+\gamma_{4}\right) .
$$

We introduce some notation. Let $\theta_{1}, \theta_{2}, \ldots$ denote independent random variables 
uniformly distributed in $[0,1]$ and independent of all other random variables considered. For a vector-valued smooth function $H$ we use the Taylor expansion

$$
H(x)=H(0)+H^{\prime}(0) x+\ldots+H^{(n)}(0) \frac{x^{n}}{n !}+\mathrm{E}_{\theta_{1}} H^{(n+1)}\left(\theta_{1} x\right)\left(1-\theta_{1}\right)^{n} \frac{x^{n+1}}{n !} .
$$

Here $\mathrm{E}_{\theta_{1}}$ denotes the conditional expectation given all the random variables but $\theta_{1}$. In particular, we have the mean value formula, $H(x)-H(0)=\mathrm{E}_{\theta_{1}} H^{\prime}\left(\theta_{1} x\right) x$.

Given a sum $S=s_{1}+\ldots+s_{k}$, denote $S^{(i)}=S-s_{i}$ and, similarly, $S^{(i, j)}=S-s_{i}-s_{j}$.

Using the fact that the distribution of $U$ coincides with the conditional distribution of

$$
\begin{aligned}
U_{0} & :=\sum_{1 \leqslant i<j \leqslant n} h\left(A_{i}, A_{j}\right) v_{i} v_{j} \\
& =\sum_{i=1}^{n} g_{1}\left(A_{i}\right)\left(v_{i}-p\right)+\sum_{1 \leqslant i<j \leqslant n} g_{2}\left(A_{i}, A_{j}\right)\left(v_{i}-p\right)\left(v_{j}-p\right),
\end{aligned}
$$

conditioned on the event $\mathbb{B}:=\left\{S_{0}=N\right\}$, where $S_{0}=\sum_{i=1}^{n} v_{i}$, we obtain

$$
\hat{F}(t)=\frac{1}{2 \pi \mathrm{P}\{\mathbb{B}\}} \int_{-\pi}^{\pi} \operatorname{Ee}\left\{t U_{0}+s\left(S_{0}-N\right)\right\} \mathrm{d} s ;
$$

see Erdös and Rényi (1959). Write

$$
\begin{aligned}
& T=\sum_{i=1}^{n} T_{i}, \quad T_{i}=z_{i}\left(v_{i}-p\right), \quad z_{i}=t x_{i}+s \tau^{-1}, \quad x_{i}=g_{1}\left(A_{i}\right), \quad \tau=(n p q)^{1 / 2}, \\
& Q=\sum_{1 \leqslant i<j \leqslant n} Q_{i, j}, \quad Q_{i, j}=t y_{i, j}\left(v_{i}-p\right)\left(v_{j}-p\right), \quad y_{i, j}=g_{2}\left(A_{i}, A_{j}\right) .
\end{aligned}
$$

We have $T+Q=t U_{0}+s \tau^{-1}\left(S_{0}-N\right)$ and, therefore,

$$
\hat{F}(t)=\lambda \int_{-\pi \tau}^{\pi \tau} \operatorname{Ee}\{T+Q\} \mathrm{d} s, \quad \lambda^{-1}=2 \pi \tau \mathrm{P}\{\mathbb{B}\} .
$$

Höglund (1978) showed that $2^{-1 / 2} \pi \leqslant \lambda^{-1} \leqslant(2 \pi)^{1 / 2}$; see (5.16). We shall approximate the integrand $\operatorname{Ee}\{T+Q\}$ by the sum $h_{1}+h_{2}$, where

$$
h_{1}=\operatorname{Ee}\{t\}, \quad h_{2}=\mathrm{i}^{3}\left(\begin{array}{l}
n \\
2
\end{array}\right) \operatorname{Ee}\left\{T^{(1,2)}\right\} V, \quad V=Q_{1,2} T_{1} T_{2} .
$$

To prove (4.1) it clearly suffices to prove the inequalities

$$
\begin{gathered}
\int_{|t| \leqslant H_{1}}\left|\lambda \int_{|s| \leqslant \pi \tau}\left(h_{1}+h_{2}\right) \mathrm{d} s-\hat{G}(t)\right| \frac{\mathrm{d} t}{|t|} \ll \mathscr{R}, \\
I:=\int_{|t| \leqslant H_{1}} \lambda \int_{|s| \leqslant \pi \tau}\left|\operatorname{Ee}\{T+Q\}-\left(h_{1}+h_{2}\right)\right| \mathrm{d} s \frac{\mathrm{d} t}{|t|} \ll \mathscr{R} .
\end{gathered}
$$

Note that in the i.i.d. case the inequality corresponding to (4.2) is proved in Lemma 6.1 of Bentkus et al. (1997). We prove (4.2) by combining the proof of this lemma with the proof of 
the Berry-Esseen bound for the finite-population sample mean given in Höglund (1978). For details we refer to Lemma 4.3 of Bloznelis and Götze (1997).

To prove (4.3), we expand e $\{T+Q\}$ in powers of $T_{i}$ and $Q_{i, j}$. In order to ensure the integrability (with respect to the measure $\mathrm{d} s \mathrm{~d} t /|t|$ ) of the remainders of these expansions we split $\operatorname{Ee}\{T+Q\}$ into a product of two functions (different for different values of $s$ and $t$ ) so that the first one is the characteristic function of a sum of conditionally independent random variables and vanishes sufficiently fast as $s$ and $t$ tend to infinity. This type of approach has been used earlier by Helmers and van Zwet (1982), van Zwet (1984), Götze and van Zwet (1991) and Bentkus et al. (1997) in the i.i.d. situation.

Introduce the set $\mathscr{Z}=\left\{(s, t):|s| \leqslant \pi \tau,|t| \leqslant H_{1}\right\}$. For technical reasons it is convenient to split the integral $I$ in two parts $I=I_{1} I_{2}$ according to the regions $\mathscr{E}=\mathscr{Z}_{1} \cup \mathscr{Z}_{2}$,

$$
Z_{1}=\mathscr{Z} \cap\left\{|t| \leqslant C_{3} q^{-1}\right\} \quad \text { and } \quad Z_{2}=\mathscr{Z} \cap\left\{C_{3} q^{-1}<|t| \leqslant H_{1}\right\} .
$$

Here $C_{3}$ denotes a sufficiently large absolute constant. We choose $C_{3}=600 \Theta^{-1}(1)$. In Lemma 4.1 we prove the bound $I_{2} \ll \mathscr{B}$. The proof of the bound $I_{2} \ll \mathscr{B}$ is similar but simpler. We skip it and refer to Lemma 4.2 Bloznelis and Götze (1997) for details. It remains to prove Lemma 4.1.

Note that, for any $i, j, i_{1}, \ldots, i_{k} \in \Omega_{n}$ such that $\{i, j\} \cap\left\{i_{1}, \ldots, i_{k}\right\}=\varnothing$ we have

$$
\mathrm{E}^{i_{1}, \ldots, i_{k}}\left|y_{i, j}\right|^{r} \leqslant c(k, r) \mathrm{E}\left|y_{i, j}\right|^{r}, \quad \mathrm{E}^{i_{1}, \ldots, i_{k}}\left|x_{j}\right|^{r} \leqslant c(k, r) \mathrm{E}\left|x_{j}\right|^{r}, \quad r \geqslant 0 .
$$

We need to introduce some more notation. Given $D=\{i, j, \ldots, k\} \subset \Omega_{n}$, let $\mathrm{E}_{\{D\}}=$ $\mathrm{E}_{\{i, j, \ldots, k\}}$ and $\mathrm{E}_{[D]}=\mathrm{E}_{[i, j, \ldots, k]}$ denote the conditional expectation given all the random variables but $\left\{v_{j}, j \in D\right\}$ and the conditional expectation given $\left\{v_{j}, A_{j}, j \in D\right\}$, respectively. Given $1 \leqslant m \leqslant n$, introduce the random variables

$$
\xi_{i}=t\left(v_{i}-p\right) \xi_{m}\left(A_{i}\right), \quad \xi_{m}(a)=\sum_{j=m+1}^{n} g_{2}\left(a, A_{j}\right)\left(v_{j}-p\right) .
$$

Here $i \in \Omega_{m}$ and $a \in \mathscr{C} \backslash\left\{A_{m+1}, \ldots, A_{n}\right\}$. Given $B \subset \Omega_{m}$, denote

$$
Y_{B}=\left|\mathrm{E}_{\{B\}} \mathrm{e}\left\{\sum_{i \in B} T_{i}\right\}\right|, Z_{B}=\mid \mathrm{E}_{\{B\}} \mathrm{e}\left\{\sum_{i \in B}\left(T_{i}+\xi_{i}\right)\right\} .
$$

Furthermore, given $A_{i}, i \in B$, let $A_{B}^{*}$ denote the random variable uniformly distributed in the set $\left\{A_{i}, i \in B\right\}$ and let $\mathrm{E}_{B}^{*}$ denote the conditional expectation given all the random variables but $A_{B}^{*}$. Introduce the random variables

$$
\Psi_{B}=g_{B}(t) \prod_{k \in B} u_{[1]}^{1 / 2}\left(z_{k}\right), \quad \kappa_{B}=\alpha N E_{B}^{*} \xi_{m}^{2}\left(A_{B}^{*}\right), \quad I_{B}=I\left\{\kappa_{B}>\delta\right\}
$$

where $\alpha=2 \pi\left(4 \Theta^{-1}(1)+1\right)$ and $\delta=\Theta(1) / 40$ are constants,

$$
g_{B}(t)=\exp \left\{p q \frac{\delta}{2} \frac{|B|}{N} t^{2}\right\}, \quad u_{[d]}(x)=1-\frac{p q}{2} \Theta(d) x^{2} I\{|x|<d+\pi\}, \quad d>0 .
$$

In Lemma 5.4 below, for $|t| \leqslant H_{1}$ and $|s| \leqslant \pi \tau$, we prove the inequalities 


$$
Z_{B} \ll I_{B}+\Psi_{B}, \quad Y_{B} \ll \Psi_{B}, \quad \mathrm{E}^{i_{1}, \ldots, i_{4}} \Psi_{B}^{r} \ll F_{B}^{r}, \quad r=1,2,
$$

where $i_{1}, \ldots, i_{4} \in \Omega_{n} \backslash B$. Here we denote

$$
F_{B}=\exp \left\{-8 \delta p q|B| N^{-1}\left(t^{2}+s^{2} / q\right)\right\} .
$$

We often take $|B| \geqslant m / 4$, with $m$ given by (4.13). In this case we have

$$
F_{B} \leqslant\left(t^{2}+s^{2} / q\right)^{-10} \text {. }
$$

Lemma 4.1. Assume that $\beta_{2}=1$ and that (3.3) holds. Then

$$
I_{2}=\lambda \int_{\mathscr{\mathscr { Z }}_{2}} \frac{\left|\operatorname{Ee}\{T+Q\}-\left(h_{1}+h_{2}\right)\right|}{|t|} \mathrm{d} s \mathrm{~d} t \ll \mathscr{B},
$$

where $\mathscr{Z}_{2}$ is given by (4.4).

Proof. Given a positive number $L$ and a complex-valued function $f(s, t)$, we write $f \prec L$ if

$$
\int_{\mathscr{Z}_{2}}|f(s, t) \| t|^{-1} \mathrm{~d} s \mathrm{~d} t \ll L .
$$

Furthermore, for two complex-valued functions $f, g$ we write $f \sim g$ if $f-g \prec \mathscr{B}$. In view of the inequality $\lambda \leqslant 2^{1 / 2} \pi^{-1}$, (4.11) can be abbreviated as follows:

$$
\operatorname{Ee}\{T+Q\} \sim h_{1}+h_{2} \text {. }
$$

Given $(s, t) \in \mathscr{Z}_{2}$ wirte $u=t^{2}+s^{2} / q$ and let

$$
m=m(s, t)>C_{4} q^{-1} n u^{-1} \ln u, \quad C_{4}=300 \Theta^{-1}(1),
$$

denote the smallest integer which is greater than $C_{4} q^{-1} n u^{-1} \ln u$. A simple calculation shows that $C_{4} \leqslant m(s, t) \leqslant C_{4} C_{3}^{-1} n$, for $(s, t) \in \mathscr{Z}_{2}$. Since $C_{4}=C_{3} / 2$ we have $10 \leqslant m(s, t) \leqslant$ $n / 2$.

Write $\mu:=m p q N^{-1}=C_{4} u^{-1} \ln u$. We shall often use the following fact,

$$
\left(t^{2}\right)^{\alpha}\left(s^{2}\right)^{\beta} \mu^{\gamma} \prec q^{\beta+1 / 2} c(\alpha, \beta, \gamma), \quad \text { for } \gamma>\alpha+\beta+\frac{1}{2}, \quad \alpha, \beta \geqslant 0 .
$$

In what follows $B$ always denote the set $\{4, \ldots, m\} . R, R_{1}, R_{2} \ldots$ will denote random variables (remainders) which may be different in different places. This will not cause any misunderstanding if we assume that $R, R_{1}, R_{2} \ldots$ always take the latest prescribed values.

Let us prove (4.12). Split $Q=Q_{A}+Q_{D}+\xi$ and $T=T_{A}+T_{D}$, where

$$
\begin{aligned}
Q_{A} & =\sum_{1 \leqslant i<j \leqslant m} Q_{i, j}, \quad Q_{D}=\sum_{m<i<j \leqslant N} Q_{i, j}, \quad \xi=\sum_{1 \leqslant i \leqslant m} \xi_{i}, \\
T_{A} & =\sum_{1 \leqslant i \leqslant m} T_{i}, \quad T_{D}=\sum_{m<i \leqslant N} T_{i},
\end{aligned}
$$

and where the $\xi_{i}$ are given by (4.6). Furthermore, write $W=T_{D}+Q_{D}$. We have $T+Q$ $=T_{A}+Q_{A}+\xi+W$ and $\mathrm{e}\{T+Q\}=v \mathrm{e}\left\{Q_{A}\right\}$, with $v=\mathrm{e}\left\{W+T_{A}+\xi\right\}$. Expanding in powers of $\mathrm{i} Q_{A}$ and using symmetry, we obtain 


$$
\mathrm{Ee}\{T+Q\}=f_{1}^{*}+f_{2}^{*}+R, \quad F_{1}^{*}=\mathrm{E} v, \quad f_{2}^{*}=\mathrm{i}\left(\begin{array}{c}
m \\
2
\end{array}\right) \operatorname{E} v Q_{1,2},
$$

with $|R| \leqslant \mathrm{E} Q_{A}^{2}$. By symmetry, we have

$$
\mathrm{E} Q_{A}^{2}=\left(\begin{array}{c}
m \\
2
\end{array}\right) p^{2} q^{2} t^{2} \mathrm{E} y_{1,2}^{2} \leqslant \mu^{2} t^{2} N^{-1} \gamma_{2} \prec \mathscr{B} .
$$

Now (4.14) implies e $\{T+Q\} \sim f_{1}^{*}+f_{2}^{*}$.

The rest of the proof consists of two steps. In the first step we show that

$$
f_{2}^{*} \sim h_{3}, \quad h_{3}=\mathrm{i}^{3}\left(\begin{array}{c}
m \\
2
\end{array}\right) \operatorname{Ee}\left\{T^{(1,2)}\right\} V .
$$

In the second step we prove

$$
f_{1}^{*} \sim h_{1}+h_{4}, \quad \text { where } h_{4}=h_{2}-h_{3} .
$$

Step 1. We start by showing

$$
f_{2}^{*} \sim f_{3}^{*}, \quad f_{3}^{*}=\mathrm{i}\left(\begin{array}{c}
m \\
2
\end{array}\right) \mathrm{E} v_{1} Q_{1,2}, \quad v_{1}=\mathrm{e}\left\{W+T_{A}+\xi^{(1,2)}\right\} .
$$

Write $v=v_{1} \mathrm{e}\left\{\xi_{1}+\xi_{2}\right\}$. expanding the exponent in powers of $\left(\xi_{1}+\xi_{2}\right)$, we obtain

$$
\begin{gathered}
f_{2}^{*}=f_{3}^{*}+f_{4}^{*}+f_{5}^{*}+f_{6}^{*}, \quad f_{j}^{*}=\mathrm{i}^{2}\left(\begin{array}{c}
m \\
2
\end{array}\right) \mathrm{E} v_{1} Q_{1,2} l_{j}, \quad j=4,5,6, \\
l_{4}=\xi_{1}+\xi_{2}, \quad l_{5}=\left(\xi_{1}^{2}+\xi_{2}^{2}\right) v_{2}, \quad l_{6}=2 \xi_{1} \xi_{2} v_{2}, \quad v_{2}=\mathrm{i}^{2} \mathrm{e}\left\{\theta_{1}\left(\xi_{1}+\xi_{2}\right)\right\}(1-\theta) .
\end{gathered}
$$

In order to prove (4.17) we shall show $f_{i}^{*} \sim 0$, for $i=4,5,6$.

To show $f_{6}^{*} \sim 0$, we bound $\left|v_{1} v_{2}\right| \leqslant 1$ and obtain

$$
\left|f_{6}^{*}\right| \leqslant m^{2} \mathrm{E}\left|Q_{1,2} \xi_{1} \xi_{2}\right|=m^{2} p^{2} q^{2}|t|^{3} \mathrm{E}\left|y_{1,2} \zeta_{m}\left(A_{1}\right) \xi_{m}\left(A_{2}\right)\right| .
$$

Combining the inequalities $\left|\xi_{m}\left(A_{1}\right) \xi_{m}\left(A_{2}\right)\right| \leqslant \xi_{m}^{2}\left(A_{1}\right)+\xi_{m}^{2}\left(A_{2}\right)$ and

$$
\mathrm{E}\left|y_{1,2}\right| \zeta_{m}^{2}\left(A_{i}\right)=p q(n-m) \mathrm{E}\left|y_{1,2}\right| y_{i, n}^{2} \leqslant q N^{-7 / 2} \gamma_{3}, \quad i=1,2,
$$

and the bound $|t| \leqslant N^{1 / 2}$, we obtain $\left|f_{6}\right| \ll \mu^{2} t^{2} \gamma_{3} N^{-1} \prec \mathscr{R}$.

Let us show $f_{5}^{*} \sim 0$. By symmetry, it suffices to show $m^{2} \mathrm{E} v_{1} v_{2} Q_{1,2} \xi_{1}^{2} \sim 0$. Expanding the exponent in $v_{2}$ in powers of $\mathrm{i} \theta \xi_{2}$ and then the exponent in $v_{1}$ in powers of $\mathrm{i} T_{2}$ we obtain

$$
\left|\mathrm{E} v_{1} v_{2} Q_{1,2} \xi_{1}^{2}\right| \leqslant R_{1}+R_{2}, \quad R_{1}=\mathrm{E}\left|Q_{1,2} \xi_{2}\right| \xi_{1}^{2}, \quad R_{2}=\mathrm{E}\left|Q_{1,2} T_{2}\right| \xi_{1}^{2}
$$

Invoking (4.45) and the inequality $|t| \leqslant N^{1 / 2}$, we obtain

$$
m^{2} R_{1} \ll \mu^{2} t^{4} N^{-5 / 2} \gamma_{4} \prec \mathscr{B}, \quad m^{2} R_{2} \prec \mu^{2} t^{2} N^{-1}\left(1+\gamma_{4}\right) \prec \mathscr{B},
$$

thus completing the proof of $f_{5}^{*} \sim 0$.

Let us show $f_{4}^{*} \sim 0$. By symmetry, it suffices to show $m^{2} R \sim 0$ with $R=\mathrm{E} v_{1} Q_{1,2} \xi_{1}$. Expanding $v_{1}$ in powers of $\mathrm{i} T_{2}$, we can replace $v_{1}$ by $\mathrm{i} T_{2} v_{3}$, with $v_{3}=\mathrm{e}\{W+$ $\left.T_{A}^{(2)}+\xi^{(1,2)}+\theta_{1} T_{2}\right\}$. Now, using the simple bound $\left|\mathrm{E}_{\{B\}} v_{3}\right| \leqslant Z_{B}$, we obtain 


$$
|R| \leqslant \mathrm{E} R_{1} R_{2}, \quad R_{1}=\left|Q_{1,2} T_{2}\right|, \quad R_{2}=\mathrm{E}_{[1,2]}\left|\xi_{1}\right| Z_{B}
$$

First we bound $R_{2}$. By Hölder's inequality,

$$
R_{2} \leqslant R_{3} R_{4}, \quad R_{3}^{2}=\mathrm{E}_{[1,2]} \xi_{1}^{2}, \quad R_{4}^{2}=\mathrm{E}_{[1,2]} Z_{B}^{2} .
$$

Furthermore, by (4.9), $R_{4}^{2} \leqslant 2 R_{5}^{2}+2 R_{6}^{2}$, where

$$
R_{5}^{2}=\mathrm{E}_{[1,2]} I_{B}^{2} \leqslant \delta^{-1} \mathrm{E}_{[1,2]} \kappa_{B} \ll \mathrm{E}_{[1,2]} \kappa_{B}, \quad R_{6}^{2}=\mathrm{E}_{[1,2]} \Psi_{B}^{2} \leqslant F_{B}^{2} .
$$

Combining (4.20) with the relations (which follow from symmetry and (4.5))

$$
\begin{aligned}
& \mathrm{E}_{[1,2]} \xi_{1}^{2}=p q(n-m)\left(v_{1}-p\right)^{2} t^{2} \mathrm{E}^{1,2} y_{1, n}^{2}=q\left(v_{1}-p\right)^{2} t^{2} N \mathrm{E}^{1,2} y_{1, n}^{2}, \\
& \mathrm{E}_{[1,2]} \kappa_{b}=\alpha N \mathrm{E}_{[1,2]} \xi_{m}\left(A_{4}\right)^{2}=\alpha N(n-m) p q \mathrm{E}^{1,2} y_{4, n}^{2} \ll q N^{-1} \gamma_{2},
\end{aligned}
$$

we obtain

$$
R_{3} R_{5} \ll q\left|\nu_{1}-p \| t\right| \gamma_{2}^{1 / 2} \tilde{\gamma}_{2}^{1 / 2} \quad \text { and } \quad R_{3} R_{6} \ll N^{1 / 2} q^{1 / 2}\left|\nu_{1}-p \| t\right| \tilde{\gamma}_{2}^{1 / 2} F_{B} .
$$

Here we denote $\tilde{\gamma}_{2}=\mathrm{E}^{1,2} y_{1, n}^{2}$. using the first inequality of (4.23), we obtain

$$
m^{2} \mathrm{E} R_{1} R_{3} R_{5} \ll m^{2} p^{2} q^{3} t^{2} \gamma_{2}^{1 / 2} \mathrm{E}\left|y_{1,2}\right| \tilde{\gamma}_{2}^{1 / 2}\left|z_{2}\right|,
$$

and invoking the second inequality of (4.47), we obtain

$$
m^{2} \mathrm{E} R_{1} R_{3} R_{5} \ll \mu^{2} t^{2} N^{-1} \gamma_{2}^{3 / 2} \prec \mathscr{R},
$$

Using the second inequality of (4.23), we obtain

$$
m^{2} \mathrm{E} R_{1} R_{3} R_{6} \ll m^{2} p^{2} q^{5 / 2} N^{1 / 2} F_{B} t^{2} \mathrm{E}\left|y_{1,2}\right| \tilde{\gamma}_{2}^{1 / 2}\left|z_{2}\right|,
$$

and invoking the first inequality of (4.47) and (4.10), we obtain

$$
m^{2} \mathrm{E} R_{1} R_{3} R_{6} \ll \mu^{2} N^{-1} F_{B} t^{2}\left(|t| q^{1 / 2}+|s|\right) \gamma_{2} \prec \mathscr{R} .
$$

Since, by (4.18) and (4.19), $|R| \ll \mathrm{E} R_{1} R_{3} R_{5}+\mathrm{E} R_{1} R_{3} R_{6}$, it follows from (4.24) and (4.25) that $m^{2} R \sim 0$.

In the next step we show that

$$
f_{3}^{*} \sim f_{7}^{*}, \quad f_{7}^{*}=\mathrm{i}^{3}\left(\begin{array}{c}
m \\
2
\end{array}\right) \mathrm{E} v_{4} V, \quad v_{4}=\mathrm{e}\left\{w+T_{A}^{(1,2)}+\xi^{(1,2)}\right\} .
$$

Substitute $v_{1}=v_{4} \mathrm{e}\left\{T_{1}+T_{2}\right\}$ in $f_{3}^{*}$. Furthermore, using the expansion

$$
\begin{aligned}
\mathrm{e}\left\{T_{1}+T_{2}\right\} & =\left(1+T_{2}+T_{2}^{2} \mathrm{e}\left\{\theta_{1} T_{2}\right\}\left(1-\theta_{1}\right)\right) \mathrm{e}\left\{T_{1}\right\} \\
& =\mathrm{e}\left\{T_{1}\right\}+T_{2}\left(1+T_{1}+T_{1}^{2} \mathrm{e}\left\{\theta_{2} T_{1}\right\}\left(1-\theta_{2}\right)\right)+T_{2}^{2} \mathrm{e}\left\{\theta_{1} T_{2}\right\}\left(1-\theta_{1}\right)\left(1+T_{1} \mathrm{e}\left\{\theta_{3} T_{1}\right\}\right),
\end{aligned}
$$

we obtain $\mathrm{E} v_{1} Q_{1,2}=\mathrm{E} v_{4} V+R_{1}+R_{2}$, with $\left|R_{i}\right| \leqslant \mathrm{E} Z_{B}\left|V T_{i}\right|, i=1,2$. Therefore, in order to prove (4.26) it remains to show $m^{2} R_{i} \sim 0$, for $i=1$, 2. By symmetry, it suffices to show $m^{2} R_{1} \sim 0$.

It follows from (4.9) and (4.22) that 


$$
\left|R_{1}\right| \leqslant \mathrm{E}\left|V T_{1}\right| \kappa_{B}+\mathrm{E}\left|V T_{1}\right| \Psi_{B} \ll\left(N^{-1} q \gamma_{2}+F_{b}\right) \mathrm{E}\left|V T_{1}\right| .
$$

Combining (4.46) and the inequalities $|t| \leqslant N^{1 / 2}$ and $|s| \leqslant(N q)^{1 / 2}$, we obtain

$$
\mathrm{E}\left|V T_{1}\right| \ll p^{2} q^{2}|t|\left(|t|+|s| q^{-1 / 2}\right) N^{-2}\left(\beta_{4}+\gamma_{4}\right)^{1 / 2} .
$$

Therefore,

$$
m^{2} N^{-1} q \gamma_{2} \mathrm{E}\left|V T_{1}\right| \ll \mu^{2}\left(t^{2}+s^{2}\right) N^{-1}\left(\beta_{4}+\gamma_{4}\right) \prec \mathscr{B} .
$$

Finally, (4.46) in combination with (4.10) yields $m^{2} F_{B} \mathrm{E}\left|V T_{1}\right| \prec \mathscr{B}$, and this inequality, in view of (4.28), completes the proof of (4.26).

Now we show

$$
f_{7}^{*} \sim f_{8}^{*}, \quad f_{8}^{*}=\mathrm{i}^{3}\left(\begin{array}{c}
m \\
2
\end{array}\right) \mathrm{E} v_{5} V, \quad v_{5}=\mathrm{e}\left\{W+T_{A}^{(1,2)}\right\},
$$

Expanding $v_{4}$ in powers of $\mathrm{i} \xi^{(1,2)}$, we obtain

$$
\mathrm{E} v_{4} V=\mathrm{E} v_{5} V+\mathrm{i} \mathrm{E} v_{5} V \xi^{(1,2)}+R, \quad \text { with }|R| \leqslant \mathrm{E}\left(\xi^{(1,2)}\right)|V| .
$$

Write $|R| \leqslant \mathrm{E}|V| \mathrm{E}_{[1,2]}\left(\xi^{(1,2)}\right)^{2}$. By symmetry and (4.5),

$$
\mathrm{E}_{[1,2]}\left(\xi^{(1,2)}\right)^{2}=p^{2} q^{2}(m-2)(n-m) t^{2} \mathrm{E}^{1,2} y_{3, n}^{2} \ll \mu q t^{2} N^{-1} \gamma_{2} .
$$

Now invoking (4.31) - see below - and the bound $|t| \leqslant N^{1 / 2}$, we obtain

$$
m^{2}|R| \ll \mu^{3} t^{2}\left(t^{2}+s^{2}\right) N^{-1} \gamma_{2}\left(\gamma_{2}+1\right) \prec \mathscr{B} .
$$

This inequality, together with (4.30), implies $f_{7}^{*} \sim f_{8}^{*}+f_{9}^{*}$, where

$$
f_{9}^{*}=\mathrm{i}^{4}\left(\begin{array}{c}
m \\
2
\end{array}\right) \mathrm{E} v_{5} V \xi^{(1,2)}=\mathrm{i}^{4}\left(\begin{array}{c}
m \\
2
\end{array}\right)(m-2) \mathrm{E} v_{5} V \xi_{3},
$$

in symmetry. In order to prove (4.29) it remains to show $f_{9}^{*} \sim 0$, to which we now turn.

Expanding $v_{5}$ in powers of $\mathrm{i} T_{3}$ we obtain $\left|\mathrm{E} v_{5} V \xi_{3}\right| \leqslant \mathrm{E} Y_{B}\left|V \xi_{3} T_{3}\right|$. Now, using (4.9), we obtain

$$
\left|f_{9}^{*}\right| \leqslant m^{3} \mathrm{E}\left|v_{5} V \xi_{3} T_{3}\right| \leqslant m^{3} F_{B} \mathrm{E}\left|V \xi_{3} T_{3}\right| \leqslant m^{3} F_{B} \mathrm{E}|V| \mathrm{E}^{1,2}\left|\xi_{3} T_{3}\right| .
$$

Finally, invoking (4.10) and the bounds (which follow from symmetry and (4.5))

$$
\begin{aligned}
& \mathrm{E}|V| \leqslant\left(\mathrm{E} Q_{1,2}^{2}\right)^{1 / 2}\left(\mathrm{E} T_{1}^{2} T_{2}^{2}\right)^{1 / 2} \leqslant p^{2} q^{2}|t|\left(t^{2}+s^{2} / q\right) N^{-5 / 2} \gamma_{2}^{1 / 2}, \\
& \mathrm{E}^{1,2}\left|\xi_{3} T_{3}\right| \leqslant\left(\mathrm{E}^{1,2} \xi_{3}^{2}\right)^{1 / 2}\left(\mathrm{E}^{1,2} T_{3}^{2}\right)^{1 / 2} \leqslant p q|t|(|t|+|s|) N^{-3 / 2} \gamma_{2}^{1 / 2},
\end{aligned}
$$

we obtain $f_{9}^{*} \prec \mathscr{R}$.

Let us show that $f_{8}^{*} \sim h_{3}$. Expanding $v_{5}$ in powers of $\mathrm{i} Q_{D}$, we obtain

$$
\mathrm{E} v_{5} V=\mathrm{E} v_{6} V+\mathrm{i} v_{6} V Q_{D}+R, \quad v_{6}=\mathrm{e}\left\{T^{(1,2)}\right\},
$$

with $|R| \leqslant \mathrm{E} Y_{B}|V| Q_{D}^{2}$. Note that, by symmetry,

$$
\mathrm{E} Y_{B}|V| Q_{D}^{2}=\left(\begin{array}{c}
n-m \\
2
\end{array}\right) t^{2} p^{2} q^{2} \mathrm{E} Y_{B}|V| y_{n-1, n}^{2}
$$


Invoking (4.9) and then using (4.5), we obtain

$$
\mathrm{E} Y_{B}|V| y_{n-1, n}^{2} \leqslant F_{B} \mathrm{E}|V| y_{n-1, n}^{2} \leqslant F_{B} N^{-3} \gamma_{2} \mathrm{E}|V|
$$

Combining (4.33) and (4.34) and then invoking (4.31) and (4.10), we obtain $m^{2} R \prec \mathscr{B}$. Now it follows from (4.32) that $f_{8}^{*} \sim h_{3}+f_{10}^{*}$, where

$$
f_{10}^{*}=\mathrm{i}^{4}\left(\begin{array}{c}
m \\
2
\end{array}\right) \mathrm{E} \boldsymbol{v}_{6} V Q_{D}=\mathrm{i}^{4}\left(\begin{array}{c}
m \\
2
\end{array}\right)(n-m) \mathrm{E} v_{6} V Q_{n-1, n},
$$

by symmetry.

We complete the proof of (4.15) by showing that $f_{10}^{*} \sim 0$. Expanding $v_{6}$ in powers of $\mathrm{i} T_{n-1}$ and $\mathrm{i} T_{N}$, we obtain $\left|\mathrm{E} v_{6} V Q_{n-1, n}\right| \ll \mathrm{E}\left|V^{*} V\right| Y_{B}$, where we denote $V^{*}=$ $T_{n-1} T_{n} Q_{n-1, n}$. Furthermore, using (4.9) and then invoking the simple inequality $\mathrm{E}^{1,2}\left|V^{*}\right| \ll \mathrm{E}\left|V^{*}\right|$, we obtain

$$
\mathrm{E}\left|V^{*} V\right| Y_{B} \ll F_{B} \mathrm{E}\left|V^{*} V\right| \ll F_{B} \mathrm{E}\left|V^{*}\right| \mathrm{E}|V|=F_{B}(\mathrm{E}|V|)^{2} .
$$

Therefore, $\left|f_{10}^{*}\right| \leqslant m^{2}(n-m) F_{B}(\mathrm{E}|V|)^{2}$. Finally, an application of (4.31) and (4.10) yields $f_{10}^{*} \prec \mathscr{R}$, thus completing the proof of (4.15).

Step 2. In order to prove (4.16) it suffices to show that

$$
\begin{aligned}
& f_{1}^{*} \sim f_{11}^{*}+f_{12}^{*}, \quad f_{11}^{*}=\mathrm{E} v_{7}, \quad f_{12}^{*}=\mathrm{iE} v_{7} \xi, \quad v_{7}=\mathrm{e}\left\{T+Q_{D}\right\}, \\
& f_{11}^{*} \sim h_{1}+f_{13}^{*}, \quad f_{13}^{*}=\left(\begin{array}{c}
n-m \\
2
\end{array}\right) \mathrm{i}^{3} \operatorname{Ee}\left\{T^{(1,2)}\right\} V, \\
& f_{12}^{*} \sim f_{14}^{*}, \quad f_{14}^{*}=m(n-M) i^{3} \operatorname{Ee}\left\{t^{(1,2)}\right\} V .
\end{aligned}
$$

We begin by expanding $v$ in powers of $\mathrm{i} \xi$, to obtain

$$
f_{1}^{*}=f_{11}^{*}+f_{12}^{*}+f_{15}^{*}, \quad \text { with } f_{15}^{*}=\mathrm{i}^{2} \mathrm{E} v_{7} \xi^{2} \mathrm{e}\left\{\theta_{1} \xi\right\}\left(1-\theta_{1}\right) .
$$

In order to prove (4.35), we shall show $f_{15}^{*} \sim 0$. Split

$$
\Omega_{m}=S_{1} \cup S_{2} \cup S_{3} \cup S_{4},
$$

with $S_{i} \cap S_{j}=\varnothing, i \neq j$, and $\left|S_{j}\right| \approx m / 4,1 \leqslant j \leqslant 4$. Split $\xi=\delta_{1}+\ldots+\delta_{4}$, where $\delta_{j}=$ $\sum_{i \in S_{j}} \xi_{i}$. We have

$$
f_{15}^{*}=\sum_{1 \leqslant j, k \leqslant 4} r_{j, k}, \quad r_{j, k}=\mathrm{i}^{2} \mathrm{E} v_{7} \delta_{j} \delta_{k} \mathrm{e}\left\{\theta_{1} \xi\right\}\left(1-\theta_{1}\right) .
$$

We shall show $r_{j, k} \sim 0$, for every $1 \leqslant j, k \leqslant 4$. By symmetry, it suffices to prove $r_{1,1} \sim 0$ and $r_{1,2} \sim 0$.

Let us show $r_{1,1} \sim 0$. expanding in powers of $\mathrm{i} \theta_{1} \delta_{2}$, we obtain

$$
\mathrm{e}\left\{\theta_{1} \xi\right\}=v_{8}+\mathrm{i} \delta_{2} v_{8} \tilde{\boldsymbol{v}}, \quad \boldsymbol{v}_{8}=\mathrm{e}\left\{\theta_{1}\left(\delta_{1}+\delta_{3}+\delta_{4}\right)\right\}, \quad \tilde{\boldsymbol{v}}=\theta_{1} \mathrm{E}_{\theta_{2}} \mathrm{e}\left\{\theta_{1} \theta_{2} \delta_{2}\right\} .
$$

Substitution of this formula gives

$$
r_{1,1}=R_{1}+R_{2}, \quad R_{1}=\mathrm{i}^{2} \mathrm{E} v_{7} v_{8} \delta_{1}^{2}\left(1-\theta_{1}\right), \quad R_{2}=\mathrm{i}^{3} \mathrm{E} v_{7} v_{8} \tilde{v} \delta_{1}^{2} \delta_{2}\left(1-\theta_{1}\right) .
$$


Similarly, expanding $v_{8}$ in powers of $\mathrm{i} \theta_{1} \delta_{3}$, we obtain $R_{2}=R_{3}+R_{4}$, where

$$
R_{3}=\mathrm{i}^{3} \mathrm{E} v_{7} v_{9} \delta_{1}^{2} \delta_{2} \tilde{v}\left(1-\theta_{1}\right), \quad v_{9}=\mathrm{e}\left\{\theta_{1}\left(\delta_{1}+\delta_{4}\right)\right\}, \quad\left|R_{4}\right| \leqslant \mathrm{E} \delta_{1}^{2}\left|\delta_{2} \delta_{3}\right| .
$$

Therefore, $\left|r_{1,1}\right| \leqslant\left|R_{1}\right|+\left|R_{2}\right|+\left|R_{3}\right|$. Furthermore, invoking the inequalities $\left|\mathrm{E}_{\left\{s_{2}\right\}} \boldsymbol{v}_{7} \boldsymbol{v}_{8}\right| \leqslant$ $Y_{S_{2}}$ and $\left|\mathrm{E}_{\left\{S_{3}\right\}} v_{7} v_{9} \tilde{\boldsymbol{v}}\right| \leqslant Y_{S_{3}}$, we obtain

$$
\left|r_{1,1}\right| \leqslant r_{1}+r_{2}+r_{3}, \quad r_{1}=\mathrm{E}_{1}^{2} Y_{S_{2}}, \quad r_{2}=\mathrm{E} \delta_{1}^{2}\left|\delta_{2}\right| Y_{S_{3}}, \quad r_{3}=\mathrm{E} \delta_{1}^{2}\left|\delta_{2} \delta_{3}\right| .
$$

Now we show $r_{i} \sim 0$, for $i=1,2,3$. Denote for brevity $m_{i}=\left|S_{i}\right|, 1 \leqslant|i| \leqslant 4$.

Let us show $r_{2} \sim 0$. By symmetry,

$$
\mathrm{E}_{\left\{S_{1}\right\}} \delta_{1}^{2}=m_{1} p q t^{2} \xi_{m}^{2}\left(A_{1}\right), \quad \mathrm{E}_{\left\{S_{2}\right\}} \delta_{2}^{2}=m_{2} p q t^{2} \xi_{m}^{2}\left(A_{i_{0}}\right)
$$

with $i_{0} \in S_{2}$. Combining (4.39) and the inequality $\mathrm{E}_{\left\{S_{2}\right\}}\left|\delta_{2}\right| \leqslant\left(\mathrm{E}_{\left\{S_{2}\right\}} \delta_{2}^{2}\right)^{1 / 2}$ and using symmetry again, we obtain

$$
\begin{aligned}
r_{2}=\mathrm{E}_{S_{3}}\left(\mathrm{E}_{\left\{S_{1}\right\}} \delta_{1}^{2}\right) \mathrm{E}_{\left\{S_{2}\right\}}\left|\delta_{2}\right| & \leqslant m_{1} m_{2}^{1 / 2}(p q)^{3 / 2}|t|^{3} \mathrm{E} \zeta_{m}^{2}\left(A_{1}\right)\left|\zeta_{m}\left(A_{i_{0}}\right)\right| Y_{S_{3}} \\
& \ll m^{3 / 2}(p q)^{3 / 2}|t|^{3} \mathrm{E}\left|\zeta_{m}\left(A_{1}\right)\right|^{3} Y_{S_{3}} .
\end{aligned}
$$

In the last step we applied (4.44) and again used symmetry. Furthermore, invoking (5.4) and using symmetry and (4.9), we obtain

$$
\mathrm{E}\left|\zeta_{m}\left(A_{1}\right)\right|^{3} Y_{S_{3}} \ll N^{1 / 2} p q(n-m) \mathrm{E}\left|y_{1, n}\right|^{3} Y_{S_{3}} \leqslant N^{-3} F_{S_{3}} \gamma_{3} .
$$

this inequality, in combination with (4.40) and (4.10), implies $r_{2} \sim 0$.

To show $r_{1} \sim 0$ we use symmetry, and apply (4.9) and (4.10):

$$
r_{1}=m_{1} t^{2} p^{2} q^{2}(n-m) \mathrm{E} y_{1, n}^{2} Y_{S_{2}} \ll t^{2} F_{S_{2}} N^{-1} \gamma_{2} \prec \mathscr{B} .
$$

To show $r_{3} \sim 0$, we first use (4.44) to obtain $r_{3} \leqslant \mathrm{E} \delta_{1}^{2} \delta_{2}^{2}+\mathrm{E} \delta_{1}^{2} \delta_{3}^{2}$ and then apply (4.48). Finally, collecting the bounds $r_{i} \prec \mathscr{R}, i=1,2,3$ in (4.38) we get $r_{1,1} \sim 0$.

Let us show $r_{1,2} \sim 0$. Expanding in powers of $\mathrm{i} \theta_{1} \delta_{3}$ and $\mathrm{i} \theta_{1} \delta_{4}$, we obtain

$$
\begin{aligned}
& \mathrm{e}\left\{\theta_{1} \xi\right\}=v_{10}+v_{10} v_{11} \mathrm{i} \theta_{1} \delta_{3}, \quad v_{10}=\mathrm{e}\left\{\theta_{1}\left(\delta_{1}+\delta_{2}+\delta_{4}\right)\right\}, \quad v_{11}=\mathrm{E}_{\theta_{2}} \mathrm{e}\left\{\theta_{1} \theta_{2} \delta_{3}\right\}, \\
& v_{10}=v_{12}+v_{12} v_{13} \mathrm{i} \theta_{1} \delta_{4}, \quad v_{12}=\mathrm{e}\left\{\theta_{1}\left(\delta_{1}+\delta_{2}\right)\right\}, \quad v_{13}=\mathrm{E}_{\theta_{3}} \mathrm{e}\left\{\theta_{1} \theta_{3} \theta_{4}\right\} .
\end{aligned}
$$

Combining these expansions, we obtain

$$
\mathrm{e}\left\{\theta_{1} \xi\right\}=v_{10}+v_{11} v_{12} \mathrm{i} \theta_{1} \delta_{3}+v_{11} v_{12} v_{13} \mathrm{i}^{2} \theta_{1}^{2} \delta_{3} \delta_{4}
$$

The last identity, in combination with the bounds $\left|\mathrm{E}_{\left\{S_{3}\right\}} v_{7} v_{10}\right| \leqslant Y_{S_{3}}$ and $\left|\mathrm{E}_{\left\{S_{4}\right\}} \boldsymbol{v}_{7} \boldsymbol{v}_{11} \boldsymbol{v}_{12}\right| \leqslant Y_{S_{4}}$, implies

$$
\begin{aligned}
r_{1,2} & \leqslant \mathrm{E}\left|\delta_{1} \delta_{2}\right| Y_{S_{3}}+\mathrm{E}\left|\delta_{1} \delta_{2} \delta_{3}\right| Y_{S_{4}}+\mathrm{E}\left|\delta_{1} \delta_{2} \delta_{3} \delta_{4}\right| \\
& \leqslant \mathrm{E} \delta_{1}^{2} Y_{S_{3}}+\mathrm{E} \delta_{2}^{2} Y_{S_{3}}+\mathrm{E} \delta_{1}^{2}\left|\delta_{2}\right| Y_{S_{4}}+\mathrm{E} \delta_{3}^{2}\left|\delta_{2}\right| Y_{S_{4}}+\mathrm{E} \delta_{1}^{2} \delta_{2}^{2}+\mathrm{E} \delta_{3}^{2} \delta_{4}^{2} .
\end{aligned}
$$

In the last step we used the simple inequality $a b \leqslant a^{2}+b^{2}$ several times. Note that the quantities in (4.42) can be bounded in the same way as $r_{1}, r_{2}$, and $r_{3}$ above in the proof of $r_{1,1} \sim 0$. Hence, $r_{1,2} \sim 0$ and this completes the proof of (4.35). 
Let us prove (4.36). Expanding $v_{7}$ in powers of $\mathrm{i} Q_{D}$, we obtain

$$
f_{11}^{*}=h_{1}+f_{16}^{*}+R, \quad f_{16}^{*}=\mathrm{i} \operatorname{Ee}\{T\} Q_{D},
$$

with $|R| \leqslant \mathrm{E} Y_{B} Q_{D}^{2}$. Furthermore, by symmetry,

$$
f_{16}^{*}=\left(\begin{array}{c}
n-m \\
2
\end{array}\right) \operatorname{iEe}\{T\} Q_{1,2} \text { and } \quad \mathrm{E}_{B} Q_{D}^{2}=\left(\begin{array}{c}
n-m \\
2
\end{array}\right) p^{2} q^{2} t^{2} \mathrm{E} Y_{B} y_{n-1, n}^{2} .
$$

Combining (4.9) and (4.10), we obtain $R \prec \mathscr{B}$ and, therefore, $f_{11}^{*} \sim h_{1}+f_{16}^{*}$.

Let us show $f_{16}^{*} \sim f_{13}^{*}$. Write $\mathrm{e}\{T\}=\mathrm{e}\left\{T^{(1,2)}\right\} \mathrm{e}\left\{T_{1}+T_{2}\right\}$ and use (4.27) to obtain

$$
\operatorname{Ee}\{T\} Q_{1,2}=\mathrm{i}^{2} \operatorname{Ee}\left\{T^{(1,2)}\right\} V+R_{1}+R_{2}, \quad \text { with }\left|R_{i}\right| \ll \mathrm{E}\left|V T_{i}\right| Y_{B} .
$$

By (4.9), $\left|R_{i}\right| \ll F_{B} \mathrm{E}\left|V T_{i}\right|$. Furthermore, invoking (4.46) and (4.10) we obtain $n^{2} R_{i} \prec \mathscr{R}$, $i=1$, 2. These bounds, together with (4.43), imply $f_{16}^{*} \sim f_{13}^{*}$, thus completing the proof of (4.36).

Let us prove (4.37). By symmetry, $f_{12}^{*}=m \mathrm{iE} v_{7} \xi_{1}$. Expanding $v_{7}$ in powers of $\mathrm{i} T_{1}$, we obtain

$$
f_{12}^{*}=f_{17}^{*}+R_{1}, \quad f_{17}^{*}=m i^{2} \operatorname{Ee}\left\{T^{(1)}+Q_{D}\right\} \xi_{1} T_{1}, \quad\left|R_{2}\right| \leqslant m \operatorname{E} Y_{B}\left|\xi_{1}\right| T_{1}^{2} .
$$

Furthermore, expanding the exponent in powers of $i Q_{D}$, we obtain

$$
f_{17}^{*}=f_{18}^{*}+R_{2}, \quad f_{18}^{*}=m i^{2} \operatorname{Ee}\left\{T^{(1)}\right\} \xi_{1} T_{1}, \quad\left|R_{2}\right| \leqslant m \operatorname{E} Y_{B}\left|\xi_{1} T_{1} Q_{D}\right| .
$$

Note, that by symmetry, $f_{18}^{*}=m(n-m) i^{2} \operatorname{Ee}\left\{T^{(1)}\right\} Q_{1,2} T_{1}$. Finally, expanding the exponent in powers of $\mathrm{i} T_{2}$, we obtain

$$
f_{18}^{*}=f_{14}^{*}+R_{3}, \quad \text { with }\left|R_{3}\right| \leqslant n(n-m) \mathrm{E} Y_{B}\left|V T_{2}\right| .
$$

Therefore in order to prove (4.37) it remains to show $R_{i} \prec \mathscr{R}$, for $i=1,2,3$.

To show $R_{1} \prec \mathscr{R}$ use the inequality $\left|\xi_{1}\right| T_{1}^{2} \leqslant \xi_{1}^{2}+T_{1}^{4}$. We obtain $\left|R_{1}\right| \leqslant R_{1,1}+R_{1,2}$, with $R_{1,1}=m \mathrm{E} Y_{B} T_{1}^{4}$ and $R_{1,2}=m \mathrm{E} Y_{B} \xi_{1}^{2}$. By (4.9) and (4.10), $R_{1,1} \prec \mathscr{R}$. Furthermore, the bound $R_{1,2} \prec \mathscr{R}$ is obtained in the same way as (4.41).

To show $R_{2} \prec \mathscr{R}$ use the inequality $\left|\xi_{1} T_{1} Q_{D}\right| \leqslant \xi_{1}^{2}+T_{1}^{2} Q_{D}^{2}$. We get $\left|R_{2}\right| \leqslant R_{2,1}+R_{2,2}$, with $R_{2,1}=m \mathrm{E} Y_{B} \xi_{1}^{2} \prec \mathscr{B}$ (cf. (4.41)) and with

$$
R_{2,2}=m \mathrm{E} Y_{B} T_{1}^{2} Q_{D}^{2}=m\left(\begin{array}{c}
n-m \\
2
\end{array}\right) \mathrm{E} Y_{B} T_{1}^{2} Q_{n-1, n}^{2} \leqslant m n^{2} F_{B} \mathrm{E} T_{1}^{2} Q_{n-1, n}^{2},
$$

by symmetry and (4.9). Now, combining (4.10) and the inequality

$$
\mathrm{E} T_{1}^{2} Q_{n-1, n}^{2}=p^{3} q^{3} t^{2} \mathrm{E}_{1}^{2} \mathrm{E}^{1} y_{n-1, n}^{2} \ll p^{3} q^{3} t^{2}\left(t^{2}+s^{2} / q\right) N^{-4} \gamma_{2},
$$

(here we use (4.5)) we obtain $R_{2,2} \prec \mathscr{R}$.

To show $R_{3} \prec \mathscr{R}$ we apply (4.9) to obtain $R_{3} \leqslant n m F_{B} \mathrm{E}\left|V T_{2}\right|$. Then combining (4.46) and (4.10) we obtain $R_{3} \prec \mathscr{B}$. We arrive at (4.37), thus completing the proof of the lemma.

In the next lemma we gather together some auxiliary inequalities used in Lemma 4.1. We shall frequently use the inequalities 


$$
a b \leqslant a^{2}+b^{2}, \quad a^{2} b \leqslant a^{3}+b^{3} .
$$

Lemma 4.2 We have

$$
\begin{aligned}
& \mathrm{E}\left|Q_{1,2} \xi_{2}\right| \xi_{1}^{2} \ll p^{2} q^{3} t^{4} N^{-9 / 2} \gamma_{4}, \quad \mathrm{E}\left|Q_{1,2} T_{2}\right| \xi_{1}^{2} \ll p^{2} q^{3}|t|^{3} N^{-7 / 2}\left(1+\gamma_{4}\right), \\
& \mathrm{E}\left|Q_{1,2} T_{2} T_{1}^{2}\right| \ll p^{2} q^{2}|t|\left(|t|^{3}+|s|^{3} q^{-3 / 2}\right) N^{-3}\left(\beta_{4}+\gamma_{4}\right)^{1 / 2}, \\
& \mathrm{E}\left|y_{1,2} x_{2}\right| \tilde{\gamma}_{2}^{1 / 2} \ll N^{-7 / 2} \gamma_{2}, \quad \mathrm{E}\left|y_{1,2} z_{2}\right| \tilde{\gamma}_{2}^{1 / 2} \ll N^{-3} \gamma_{2}, \quad \tilde{\gamma}_{2}=\mathrm{E}^{1,2} y_{1, n}^{2}, \\
& \mathrm{E} \delta_{K}^{2} \delta_{M}^{2} \ll p^{2} q^{2} t^{2} m^{2} N^{-3} \gamma_{4}, \quad \text { for any } K, M \subset \Omega_{m}, K \cap M=\varnothing,
\end{aligned}
$$

with $|K|,|M|>0$. Here $\delta_{K}=\sum_{i \in K} \xi_{i}$.

Proof. Let us prove (4.45). We have

$$
\mathrm{E}\left|Q_{1,2} \xi_{2} \delta_{1}^{2}\right| \ll p^{2} q^{2} t^{4} \mathrm{E}\left|y_{1,2} \zeta_{m}\left(A_{2}\right)\right| \zeta_{m}^{2}\left(A_{1}\right) \leqslant 2 p^{2} q^{2} t^{4} \mathrm{E}\left|y_{1,2} \zeta_{m}^{3}\left(A_{1}\right)\right|,
$$

where in the last step we apply (4.44) and use symmetry. Furthermore, writing $\mathrm{E}\left|y_{1,2} \zeta_{m}^{3}\left(A_{1}\right)\right|=\mathrm{E}\left|y_{1,2}\right| \mathrm{E}^{1,2}\left|\xi_{m}^{3}\left(A_{1}\right)\right|$ and invoking (5.4), we obtain the first inequality in (4.45). To prove the second inequality we apply (4.21),

$$
\mathrm{E}\left|Q_{1,2} T_{2}\right| \xi_{1}^{2}=\mathrm{E}\left|Q_{1,2} T_{2}\right| \mathrm{E}_{[1,2]} \xi_{1}^{2} \leqslant p^{2} q^{3} N|t|^{3} \mathrm{E} y_{1, n}^{2}\left|y_{1,2} z_{2}\right|,
$$

and use the inequality $\mathrm{E} y_{1, n}^{2}\left|y_{1,2} z_{2}\right| \leqslant N^{-9 / 2}\left(1+\gamma_{4}\right)$. To prove this inequality combine (4.5), Hölder's inequality and the bounds

$$
\left|y_{1,2} z_{2}\right| \leqslant \sqrt{N}\left|y_{1,2} x_{2}\right|+\left|y_{1,2}\right|, \quad\left|y_{1,2} x_{2}\right| \leqslant N y_{1,2}^{2}+N^{-1} x_{2}^{2} .
$$

Let us prove (4.46). We have $\mathrm{E}\left|Q_{1,2} T_{2}\right| T_{1}^{2} \leqslant p^{2} q^{2}|t| \mathrm{E}\left|y_{1,2} z_{2}\right| z_{1}^{2}$. Now (4.46) follows from the bound

$$
\mathrm{E}\left|y_{1,2} z_{2}\right| z_{1}^{2} \leqslant\left(|t|^{3}+t^{2}|s| q^{-1 / 2}+|t| s^{2} / q+|s|^{3} q^{-3 / 2}\right) N^{-3}\left(\beta_{4}+\gamma_{4}\right)^{1 / 2}
$$

which is a consequence of the following inequalities:

$$
\begin{gathered}
z_{1}^{2}\left|z_{2}\right| \leqslant\left(t^{2} x_{1}^{2}+s^{2} / \tau^{2}\right)\left|z_{2}\right|, \quad \mathrm{E}\left|y_{1,2} x_{2}\right| \leqslant\left(\mathrm{E} y_{1,2}^{2}\right)^{1 / 2}\left(\mathrm{E} x_{2}^{2}\right)^{1 / 2} \leqslant N^{-2} \gamma_{2}^{1 / 2}, \\
\mathrm{E}\left|y_{1,2} x_{2}\right| x_{1}^{2} \leqslant\left(\mathrm{E} y_{1,2}^{2} x_{1}^{2}\right)^{1 / 2}\left(\mathrm{E} x_{1}^{2} x_{2}^{2}\right)^{1 / 2} \leqslant N^{-1}\left(\mathrm{E} y_{1,2}^{4} \mathrm{E} x_{1}^{4}\right)^{1 / 4} \leqslant N^{-3}\left(\beta_{4}+\gamma_{4}\right)^{1 / 2}, \\
\mathrm{E}\left|y_{1,2}\right| x_{1}^{2} \leqslant\left(\mathrm{E} y_{1,2}^{2} x_{1}^{2}\right)^{1 / 2}\left(\mathrm{E} x_{1}^{2}\right)^{1 / 2} \leqslant N^{-1 / 2}\left(\mathrm{E} y_{1,2}^{4} \mathrm{E} x_{1}^{4}\right)^{1 / 2} \leqslant N^{-5 / 2}\left(\beta_{4}+\gamma_{4}\right)^{1 / 2} .
\end{gathered}
$$

Let us prove (4.47). By (4.44), $\left|y_{1,2} x_{2}\right| \tilde{\gamma}_{2}^{1 / 2} \leqslant N^{-1 / 2} y_{1,2}^{2}+N^{1 / 2} x_{2}^{2} \tilde{\gamma}_{2}$. Now invoking (4.5), we obtain the first inequality of (4.47). To prove the second one, write

$$
\mathrm{E}\left|y_{1,2} z_{1}\right| \tilde{\gamma}_{2}^{1 / 2} \leqslant N^{1 / 2} \mathrm{E}\left|y_{1,2} x_{2}\right| \tilde{\gamma}_{2}^{1 / 2}+\mathrm{E}\left|y_{1,2}\right| \tilde{\gamma}_{2}^{1 / 2}
$$

(where we have used the bounds $|t| \leqslant N^{1 / 2}$ and $|s| \leqslant \tau$ ) and apply Hölder's inequality to the second summand.

Let us prove (4.48). By symmetry, 


$$
\mathrm{E} \delta_{K}^{2} \delta_{M}^{2}=|K \| M| p^{2} q^{2} t^{4} \mathrm{E} \xi_{m}^{2}\left(A_{1}\right) \xi_{m}^{2}\left(A_{2}\right)
$$

A simple calculation yields

$$
\mathrm{E} \zeta_{m}^{2}\left(A_{1}\right) \xi_{m}^{2}\left(A_{2}\right) \leqslant p q(n-m) N^{-6} \gamma_{4}+p^{2} q^{2}(n-m)(n-m-1) N^{-6} \gamma_{2}^{2} \ll N^{-4} \gamma_{4}
$$

Substituting this bound in (4.49) and using the inequalities $|K|,|M|<m$ and $t^{2} \leqslant N$, we obtain (4.48).

\section{Auxiliary results}

Lemma 5.1. For the random variables $v_{i}$ and $\Theta_{i}, i=1,2,3$, defined in (3.6) and (3.25) above, the following inequalities hold:

$$
\begin{gathered}
\mathrm{E}_{i}^{2} \leqslant N^{-2} \gamma_{2}, \quad i=1,2,3, \\
\mathrm{E}^{*}\left|v_{i}\left(A_{0}^{*}\right)\right| \leqslant q^{-1} \Theta_{i}, \quad i=1,2 .
\end{gathered}
$$

For $\Lambda_{m}=\sum_{1 \leqslant i<j \leqslant m} g_{2}\left(A_{i}, A_{j}\right)$, with $3 \leqslant m \leqslant n$, we have

$$
\mathrm{E} \Lambda_{m}^{2}=\frac{m(m-1)}{2 N^{3}}\left(1-c_{\Lambda}\right) \gamma_{2}, \quad c_{\Lambda}=\frac{2(m-2)}{n-2}-\frac{(m-2)(m-3)}{(n-2)(n-3)} .
$$

For the random variable $\zeta_{m}\left(A_{k}\right)$ defined in (4.6), the inequality

$$
\mathrm{E}_{\{m+1, \ldots, n\}}\left|\zeta_{m}\left(A_{k}\right)\right|^{3} \ll p q(n p q)^{1 / 2} \sum_{j=m+1}^{n}\left|g_{2}\left(A_{k}, A_{j}\right)\right|^{3}, \quad K \leqslant m \leqslant n,
$$

holds; recall the definition of $\mathrm{E}_{\{m+1, \ldots, n\}}$ just before (4.6).

Proof. We shall prove (5.1) for case $i=1$ only. For $i=2,3$ the proof is similar. By Hölder's inequality,

$$
\Theta_{1}^{2} \leqslant \mathrm{E}^{*} v_{1}^{2}\left(A_{1}^{*}\right)=\sum_{k+1 \leqslant i, j \leqslant N} \mathrm{E}^{*} g_{2}\left(A_{1}^{*}, A_{i}\right) g_{2}\left(A_{1}^{*}, A_{j}\right)
$$

By symmetry,

$$
\mathrm{EE}^{*} g_{2}\left(A_{1}^{*}, A_{i}\right) g_{2}\left(A_{1}^{*}, A_{j}\right)=\mathrm{E} g_{2}\left(A_{1}, A_{i}\right) g_{2}\left(A_{1}, A_{j}\right)
$$

and therefore,

$$
\mathrm{E}_{1}^{2} \leqslant(N-k) \mathrm{E}_{2}^{2}\left(A_{1}, A_{2}\right)+(N-k)(N-k-1) \mathrm{E} g_{2}\left(A_{1}, A_{2}\right) g_{2}\left(A_{1}, A_{3}\right) .
$$

Now, invoking the identity

$$
\mathrm{E} g_{2}\left(A_{1}, A_{2}\right) g_{2}\left(A_{1}, A_{3}\right)=-(n-2)^{-1} \mathrm{E}_{2}^{2}\left(A_{1}, A_{2}\right),
$$

(use (1.2)) we complete the proof of $\mathrm{E}_{1}^{2} \leqslant N^{-2} \gamma_{2}$.

To prove (5.2) we combine the obvious inequality $\left|\mathscr{J}_{i}\right| /\left|\mathscr{J}_{0}\right| \leqslant q^{-1}$ and the inequalities 


$$
\mathrm{E}^{*}\left|v_{i}\left(A_{0}^{*}\right)\right|=\left|\mathscr{J}_{0}\right|^{-1} \sum_{k \in \mathscr{J}_{0}}\left|v_{i}\left(A_{k}\right)\right| \leqslant\left|\mathscr{J}_{0}\right|^{-1} \sum_{k \in \mathscr{F}_{i}}\left|v_{i}\left(A_{k}\right)\right| \leqslant \Theta_{i} \frac{\left|\mathscr{Z}_{i}\right|}{\left|\mathscr{J}_{0}\right|}, \quad i=1,2 .
$$

Let us prove (5.3). By symmetry, $\mathrm{E} \Lambda_{m}^{2}=2^{-1}(m-1) m \mathrm{E} g_{2}\left(A_{1}, A_{2}\right) \Lambda_{m}$. Furthermore,

$$
\begin{aligned}
\mathrm{E}_{2}\left(A_{1}, A_{2}\right) \Lambda_{m}= & \mathrm{E}_{2}^{2}\left(A_{1}, A_{2}\right)+2(m-2) \mathrm{E} g_{2}\left(A_{1}, A_{2}\right) g_{2}\left(A_{1}, A_{3}\right) \\
& +2^{-1}(m-2)(m-3) \mathrm{E} g_{2}\left(A_{1}, A_{2}\right) g_{2}\left(A_{3}, A_{4}\right) .
\end{aligned}
$$

Now, invoking (5.5) and the identity

$$
\mathrm{Eg}_{2}\left(A_{1}, A_{2}\right) g_{2}\left(A_{3}, A_{4}\right)=2(n-2)^{-1}(n-3)^{-1} \mathrm{Eg}_{2}^{2}\left(A_{1}, A_{2}\right)
$$

(use (1.2)) we obtain (5.3).

In order to prove (5.4) we apply Rosenthal's inequality,

$$
\mathrm{E}\left|Z_{1}+\ldots+Z_{j}\right|^{r} \leqslant c(r) \sum_{l=1}^{j} \mathrm{E}\left|Z_{l}\right|^{r}+c(r)\left(\sum_{l=1}^{j} \mathrm{E} Z_{l}^{2}\right)^{r / 2}, \quad r \geqslant 2,
$$

where $Z_{1} \ldots, Z_{j}$ are independent and centred ransom variables. We apply this inequality to the sum $\zeta_{m}\left(A_{k}\right)$ - cf. (4.15) - conditionally given $\bar{A}$,

$$
\mathrm{E}_{\{m+1, \ldots, n\}}\left|\zeta_{m}\left(A_{k}\right)\right|^{3} \ll p q \sum_{l=m+1}^{n}\left|g_{2}\left(A_{k}, A_{l}\right)\right|^{3}+\left(p q \sum_{l=m+1}^{n} g_{2}^{2}\left(A_{k}, A_{l}\right)\right)^{3 / 2} .
$$

Finally, using Hölder's inequality, we bound the second sum above by

$$
\left(\sum_{l=m+1}^{n} g_{2}^{2}\left(a, A_{l}\right)\right)^{3 / 2} \leqslant(n-m)^{1 / 2} \sum_{l=m+1}^{n}\left|g_{2}\left(a, A_{l}\right)\right|^{3}, \quad a \in \mathscr{C},
$$

thus arriving at (5.4).

Lemma 5.2. For each $0<d<\pi$ and $x, y \in \mathbb{R}$, and $\beta(x)$ defined in Section 3.1, we have

$$
|\beta(x+y)|^{2} \leqslant u_{[d]}(x) v_{[d]}(y), \quad \text { where } v_{[d]}(y)=1+p q \frac{2 \pi}{d}\left(\frac{4}{\Theta(d)}+1\right) y^{2},
$$

and where the function $u_{[d]}$ id defined in (4.8).

Proof. In the case where $|x| \geqslant \pi+d$, we have $u_{[d]}(x)=1$ and the desired inequality follows from the simple bound $|\beta(x+y)| \leqslant 1$.

In the case where $|x|<\pi+d$, we apply the mean value theorem to obtain

$$
\begin{aligned}
|\cos (x+y)-\cos (x)| & \leqslant\left|\mathrm{E} \sin \left(x+\theta_{1} y\right) y\right| \\
& \leqslant(|x|+|y|)|y| \leqslant c x^{2}+\left(c^{-1}+1\right) y^{2} .
\end{aligned}
$$

In the last step we applied the inequality $|x y| \leqslant c x^{2}+c^{-1} y^{2}$, with $c>0$. Combining (5.6) and the indentity $|\beta(x+y)|^{2}=1-2 p q(1-\cos (x+y))$, we obtain 


$$
|\beta(x+y)|^{2} \leqslant 1-2 p q\left(1-\cos (x)-c x^{2}-\left(c^{-1}+1\right) y^{2}\right) .
$$

Now invoking (5.15), we obtain

$$
|\beta(x+y)|^{2} \leqslant w_{1}+w_{2}, \quad w_{1}=1-p q(\Theta(d)-2 c) x^{2}, \quad w_{2}=\left(c^{-1}+1\right) 2 p q y^{2} .
$$

But $1-p q x^{2} \Theta(d) \geqslant d / \pi$, for $|x| \leqslant \pi+d$. Hence, $w_{1}>d / \pi$ and therefore $w_{1}+w_{2} \leqslant$ $w_{1}\left(1+\pi d^{-1} w_{2}\right)$. Choosing $c=\Theta(d) / 4$ completes the proof of the lemma.

Lemma 5.3. Assume that $\beta_{2}=1$. For every $s, t \in \mathbb{R}$ and $0<d<\pi$, we have

$$
\mathrm{E} Z^{2}\left(A_{1}\right) I_{[d]}\left(A_{1}\right) \geqslant\left(\frac{t^{2}}{N}+s^{2}\right)\left(1-2 c_{[d]}\right), \quad c_{[d]}=\max \left\{\frac{b_{1}}{d} ; \frac{b_{1}^{2}}{d^{2}}\right\} .
$$

Here $Z(a)=\operatorname{tg}_{1}(a)+s$ and $I_{[d]}(a)=I\left\{H_{1}\left|g_{1}(a)\right|<d\right\}$, for $a \in \mathscr{A}$.

Remark. A similar inequality was used by Höglund (1978), where the constatnt (corresponding to $c_{[d]}$ ) was not specified. For our purposes the dependence of $c_{d}$ on the parameters $b_{1}$ and $d$ is important and thus we include the proof.

Proof. Denote $\mathscr{K}_{[d]}=\left\{k: I_{[d]}\left(a_{k}\right)=0\right\}$. Clearly, for $r>0$,

$$
\left|\mathscr{K}_{[d]}\right|=\sum_{k \in \mathscr{K}[d]} 1 \leqslant \sum_{k \in \mathscr{K}[d]}\left|g_{1}\left(a_{k}\right) H_{1} / d\right|^{r} \leqslant n \beta_{r} \beta_{3}^{-r} b_{1}^{r} d^{-r}
$$

Furthermore, since $\mathrm{E} Z^{2}\left(A_{1}\right)=t^{2} N^{-1}+s^{2}$, we have

$$
\mathrm{E} Z^{2}\left(A_{1}\right)^{2} I_{[d]}\left(A_{1}\right)=\frac{t^{2}}{N}+s^{2}-W n^{-1}, \quad W=\sum_{k \in \mathscr{K}[d]} Z^{2}\left(a_{k}\right) .
$$

The inequality $(a+b)^{2} \leqslant 2 a^{2}+2 b^{2}$ implies $W \leqslant 2 W_{1}+2 W_{2}$, where

$$
W_{1}=s^{2}\left|K_{[d]}\right|, \quad W_{2}=t^{2} \sum_{k \in \mathscr{K}_{[d]}} g_{1}^{2}\left(a_{k}\right) \leqslant \frac{t^{2}}{N} n^{2 / 3} \beta_{3}^{2 / 3}\left|\mathscr{K}_{[d]}\right|^{1 / 3} .
$$

In the last step we applied Hölder's inequality to obtain

$$
\sum_{k \in \mathscr{K}_{[d]}} g_{1}^{2}\left(a_{k}\right) \leqslant\left(\sum_{k \in \mathscr{K}[d]}\left|g_{1}^{3}\left(a_{k}\right)\right|\right)^{2 / 3}\left|\mathscr{K}_{[d]}\right|^{1 / 3} .
$$

Now, (5.7) (with $r=2$ ) implies $W_{1} \leqslant s^{2} n c_{d}$. Furthermore, (5.7) (with $r=3$ ) implies $W_{2} \leqslant t^{2} N^{-1} n c_{d}$. These inequalities, combined with (5.8), complete the proof.

Lemma 5.4. Assume that $\beta_{2}=1$ and that (3.3) holds. For $|t| \leqslant H_{1}$ and $|s| \leqslant \pi \tau$, the inequalities (4.9) hold true.

Proof. Throughout this proof we use the notation introduced in Section 4. Fix $B \subset \Omega_{m}$. By Lemma 5.2, 


$$
Z_{B}=\prod_{k \in B}\left|\beta\left(z_{k}+t \xi_{m}\left(A_{k}\right)\right)\right| \leqslant \eta_{1} \eta_{2}, \quad \eta_{1}^{2}=\prod_{k \in B} u_{[1]}\left(z_{k}\right), \quad \eta_{2}^{2}=\prod_{k \in B} v_{[1]}\left(t \xi_{m}\left(A_{k}\right)\right) .
$$

Using the inequality $1+x \leqslant \exp \{x\}$, we obtain $\eta_{2}^{2} \leqslant \exp \left\{p q t^{2} \kappa_{B}|B| / N\right\}$ and therefore, $\left(1-I_{B}\right) \eta_{2} \leqslant g_{B}(t)$. Finally, combining the inequalities $Z_{B} \leqslant 1$ and $Z_{B} \leqslant \eta_{1} \eta_{2}$, we obtain

$$
Z_{B}=I_{B} Z_{B}+\left(1-I_{B}\right) Z_{B} \leqslant I_{B}+\left(1-I_{B}\right) \eta_{1} \eta_{2} \leqslant I_{B}+\Psi_{B},
$$

thus proving the first inequality in (4.9). Here the random variables $\Psi_{B}=\eta_{1} g_{B}(t), \kappa_{B}$ and $I_{B}$ are defined in (4.7) and the function $g_{B}(t)$ is given by (4.8).

Clearly, Lemma 5.2 (with $x=z_{k}$ and $y=0$ ) implies the second inequality of (4.9).

To prove the third and last one, observe that by Hölder's inequality we have $\mathrm{E}^{i_{1}, \ldots, i_{4}} \Psi_{B} \ll\left(\mathrm{E}^{i_{1}, \ldots, i_{4}} \Psi_{B}^{2}\right)^{1 / 2}$ and thus, it suffices to show

$$
\mathrm{E}^{i_{1}, \ldots, i_{4}} \Psi_{B}^{2} \leqslant F_{B}^{2}, \quad \text { for every } i_{1}, \ldots, i_{4} \in \Omega_{n} \backslash B .
$$

To prove (5.9) note that the inequalities $|t| \leqslant H_{1}$ and $|s| \leqslant \pi \tau$ imply

$$
u_{[1]}\left(z_{k}\right) \leqslant w\left(A_{k}\right), \quad w\left(A_{k}\right)=1-\frac{p q}{2} \Theta(1) z_{k}^{2} I_{[1]}\left(A_{k}\right), \quad k \in \Omega_{n},
$$

where we denote $I_{[1]}(a)=I\left\{H_{1}\left|g_{1}(a)\right|<1\right\}$. Therefore,

$$
\Psi_{B}^{2}=g_{B}^{2}(t) \eta_{1}^{2} \leqslant g_{B}^{2}(t) \eta, \quad \text { where } \eta=\prod_{k \in B} w\left(A_{k}\right) .
$$

Denote $D_{1}=\left\{i_{1}, \ldots, i_{4}\right\}$ and $D_{2}=\Omega_{n} \backslash D_{1}$. By Theorem 4 of Hoeffding (1963),

$$
\mathrm{E}^{i_{1}, \ldots, i_{4}} \eta \leqslant w_{*}^{|B|}, \quad \text { where } w_{*}=1-\frac{p q}{2} \Theta(1) \Gamma_{*}, \quad \Gamma_{*}=\frac{1}{\left|D_{2}\right|} \sum_{k \in D_{2}} z_{k}^{2} I_{[1]}\left(A_{k}\right) .
$$

Below we construct the following lower bound for $\Gamma_{*}$,

$$
\Gamma_{*} \geqslant \frac{9}{10}\left(t^{2}+\frac{s^{2}}{q}\right) \frac{1}{N} .
$$

Combining (5.11), (5.12) and the inequality $1+x \leqslant \exp \{x\}$ we obtain $\eta \leqslant$ $\exp \left\{-0.45 p q \Theta(1)\left(t^{2}+s^{2} / q\right)|B| N^{-1}\right\}$. Now (5.9) follows from (5.10).

Let us prove (5.12). We have

$$
\Gamma_{*}=\frac{n}{n-4} \mathrm{E} z_{1}^{2} I_{[1]}\left(A_{1}\right)-\frac{1}{n-4} M, \quad M=\sum_{k \in D_{1}} z_{k}^{2} I_{[1]}\left(A_{k}\right) .
$$

The simple inequality $(a+b)^{2} \leqslant 2 a^{2}+2 b^{2}$ gives

$$
M \leqslant 8 s^{2} / \tau^{2}+2 t^{2} M_{1}, \quad M_{1}=\sum_{k \in D_{1}} g_{1}^{2}\left(A_{k}\right) .
$$

By Hölder's inequality and (3.3), 


$$
M_{1} \leqslant 4^{1 / 3}\left(\sum_{k \in D_{1}}\left|g_{1}\left(A_{k}\right)\right|^{3}\right)^{2 / 3} \leqslant 4^{1 / 3} \beta_{3}^{2 / 3} n^{2 / 3} N^{-1} \leqslant\left(4 c_{0}\right)^{1 / 3} \frac{n}{N} .
$$

Here we have estimated $\beta_{3}^{2} / n \leqslant \beta_{4} / n \leqslant c_{0}$; see (3.3). This inequality, in combination with (5.14) implies $M \leqslant 100^{-1} n\left(t^{2}+s^{2} / q\right) N^{-1}$, provided that $c_{0}$ in (3.3) is sufficiently small. Substituting this bound in (5.13) and invoking Lemma 5.3, we obtain (5.12) thus completing the proof of the lemma.

We have used, but not so far stated Hoeffding's (1963) Theorem 4. Consider a population $\mathscr{P}$ of $n$ numbers $p_{1}, \ldots, p_{n}$. Let $\mathscr{C}_{1}, \ldots, \mathscr{C}_{\mathrm{N}}$ denote a random sample without replacement from $\mathscr{P}$ and let $\mathscr{H}_{1}, \ldots, \mathscr{H}_{N}$ denote a random sample with replacement from $\mathscr{P}$. In particular, $\mathscr{H}_{1}, \ldots, \mathscr{H}_{N}$ are independent random variables.

Theorem (Hoeffding 1963). If the function $f(x)$ is continuous and convex then

$$
\mathrm{E} f\left(\sum_{k=1}^{N} \mathscr{B}_{k}\right) \leqslant \mathrm{E} f\left(\sum_{k=1}^{N} \mathscr{Y}_{k}\right) \text {. }
$$

We conclude this paper by stating two inequalities proved by Höglund (1978):

$$
\begin{array}{cc}
1-\cos v \geqslant \frac{1}{2} v^{2} \Theta(u), \quad \text { for }|v| \leqslant \pi+u \quad \text { and } \quad 0 \leqslant u \leqslant \pi, \\
\frac{\pi^{1 / 2}}{2} \leqslant\left(\begin{array}{c}
n \\
N
\end{array}\right) s^{N}(1-s)^{n-N}(2 \pi s(1-s) n)^{1 / 2} \leqslant 1, \quad \text { with } s=\frac{N}{n},
\end{array}
$$

where $1 \leqslant N \leqslant n$.

\section{Acknowledgements}

The research for this paper was supported by Sonderforschungsbereich 343 in Bielefeld. The authors would like to thank V. Bentkus for discussions and comments.

\section{References}

Albers, W., Bickel, P.J. and van Zwet, W.R. (1976) Asymptotic expansions for the power of distribution free tests in the one-sample problem. Ann. Statist., 4, 108-156.

Babu, G.J. and Singh, K. (1985) Edgeworth expansions for sampling without replacement from finite populations. J. Multivariate Anal., 17, 261-278.

Bentkus, V., Götze, F. and van Zwet, W.R. (1997) An Edgeworth expansion for symmetric statistics. Ann. Statist., 25, 851-896.

Bickel, P.J. (1974) Edgeworth expansions in nonparametric statistics. Ann. Statist., 2, 1-20.

Bickel, P.J. and Robinson, J. (1982) Edgeworth expansions and smoothness. Ann. Probab., 10, 500-503. 
Bickel, P.J. and van Zwet, W.R. (1978) Asymptotic expansions for the power of distribution free tests in the two-sample problem. Ann. Statist., 6, 937-1004.

Bickel, P.J., Götze, F. and van Zwet, W.R. (1986) The Edgeworth expansion of $U$-statistics of degree two. Ann. Statist., 14, 1463-1484.

Bikelis, A. (1969) On the estimation of the remainder term in the central limit theorem for samples from finite populations. Studia Sci. Math. Hungar., 4, 345-354 [in Russian].

Bloznelis, M. and Götze, F. (1997) An Edgeworth expansion for finite population $U$-statistics (1997). Preprint 97-012, SFB 343, Universität Bielefeld. Publication available on the Internet at http:// www.mathematik.uni-bielefeld.de/sfb343/Welcome.html.

Bolthausen, E. and Götze, F. (1993) The rate of convergence for multivariate sampling statistics. Ann. Statist., 21, 1692-1710.

Callaert, H., Janssen, P. and Veraverbeke, N. (1980) An Edgeworth expansion for $U$-statistics. Ann. Statist., 8, 299-312.

Does, R.J.M.M. (1983) An Edgeworth expansion for simple linear rank statistics under the nullhypothesis. Ann. Statist., 11, 607-624.

Erdós, P. and Rényi, A. (1959) On the central limit theorem for samples from a finite population. Publ. Math. Inst. Hungar. Acad. Sci., 4, 49-61.

Götze, F. (1979) Asymptotic expansions for bivariate von Mises functionals. Z. Wahrscheinlichkeitstheorie verw. Geb., 50, 333-355.

Götze, F and van Zwet, W.R. (1991) Edgeworth expansions for asymptotically linear statistics. Preprint 91-034, SFB 343, Universität Bielefeld.

Helmers, R. and van Zwet, W.R. (1982) The Berry-Esseen bound for $U$-statistics. In S.S. Gupta and J.O. Berger (eds), Statistical Decision Theory and Related Topics III, Vol. 1, pp. 497-512. New York: Academic Press.

Hoeffding, W. (1963) Probability inequalities for sums of bounded random variables. J. Amer. Statist. Assoc., 58, 13-30.

Höglund, T. (1978) Sampling from a finite population: a remainder term estimate. Scand. J. Statist., 5, $69-71$.

Kokic, P.N. and Weber, N.C. (1990) An Edgeworth expansion for $U$-statistics based on samples from finite populations. Ann. Probab., 18, 390-404.

Kokic, P.N. and Weber, N.C. (1991) Rates of strong convergence for $U$-statistics in finite populations. J. Austral. Math. Soc. Ser. A, 50, 468-480.

Nandi, H.K. and Sen, P.K. (1963) On the properties of $U$-statistics when the observations are not independent II. Unbiased estimation of the parameters of a finite population. Calcutta Statist. Assoc. Bull., 12, 124-148.

Prawitz, H. (1972) Limits for a distribution, if the characteristic function is given in a finite domain. Skand. Aktuar. Tidskr., 5, 138-154.

Robinson, J. (1978) An asymptotic expansion for samples from a finite population. Ann. Statist., 6, $1005-1011$.

Serfling, R.J. (1980) Approximation Theorems of Mathematical Statistics. New York: Wiley.

Schneller, W. (1989) Edgeworth expansions for linear rank statistics. Ann. Statist., 17, 1103-1123.

van Zwet, W.R. (1982) On the Edgeworth expansion for the simple linear rank statistic. In B.V. Gnedenko, M.L. Puri and I. Vincze (eds), Nonparametric Statistical Inference, Budapest 1980, Vol. II, Colloq. Math. Soc. János Bolyai 32, pp. 889-909. Amsterdam: NorthHolland.

van Zwet, W.R. (1984) A Berry-Esseen bound for symmetric statistics. Z. Wahrscheinlichkeitstheorie verw. Geb., 66, 425-440. 
Zhao, L.C. and Chen, X.R. (1987) Berry-Esseen bounds for finite-population $U$-statistics. Sci. Sinica Ser. $A$, 30, 113-127.

Zhao, L.C. and Chen, X.R. (1990) Normal approximation for finite-population U-statistics. Acta Math. Appl. Sinica, 6, 263-272.

Received November 1997 and revised June 1999 